\title{
Optimal Coordinated Design of Multiple Damping Controllers Based on PSS and UPFC Device to Improve Dynamic Stability in the Power System
}

\author{
A. N. Hussain, F. Malek, M. A. Rashid, L. Mohamed, and N. A. Mohd Affendi \\ CERE, School of Electrical Systems Engineering, University Malaysia Perlis, 01000 Perlis, Malaysia \\ Correspondence should be addressed to A. N. Hussain; alinasser1974@yahoo.com
}

Received 28 December 2012; Accepted 19 February 2013

Academic Editor: Sebastian Anita

Copyright (c) 2013 A. N. Hussain et al. This is an open access article distributed under the Creative Commons Attribution License, which permits unrestricted use, distribution, and reproduction in any medium, provided the original work is properly cited.

Unified Power Flow Controller (UPFC) device is applied to control power flow in transmission lines. Supplementary damping controller can be installed on any control channel of the UPFC inputs to implement the task of Power Oscillation Damping (POD) controller. In this paper, we have presented the simultaneous coordinated design of the multiple damping controllers between Power System Stabilizer (PSS) and UPFC-based POD or between different multiple UPFC-based POD controllers without PSS in a singlemachine infinite-bus power system in order to identify the design that provided the most effective damping performance. The parameters of the damping controllers are optimized utilizing a Chaotic Particle Swarm Optimization (CPSO) algorithm based on eigenvalue objective function. The simulation results show that the coordinated design of the multiple damping controllers has high ability in damping oscillations compared to the individual damping controllers. Furthermore, the coordinated design of UPFC-based POD controllers demonstrates the superiority over the coordinated design of PSS and UPFC-based POD controllers for enhancing greatly the stability of the power system.

\section{Introduction}

When large power systems are interconnected through weak tie lines, Low Frequency Oscillations (LFO) in the range of $0.1-3 \mathrm{~Hz}$ are observed. These oscillations may sustain and grow to cause system separation if no adequate damping is available [1].

The installation of a Power System Stabilizer (PSS) appears as a simple and inexpensive technique for many years to produce an amount of damping torque through the injection of a supplementary stabilizing signal at a voltage reference input of an Automatic Voltage Regulator (AVR), which has increased the stability of the power system. However, their performance deteriorated when the system operating conditions varied widely [2-4]. Hence, in order to operate power systems effectively without lessening the system safety and quality of supply, even in the case of emergency conditions such as loss of transmission lines and/or generating units, new control strategies need to be applied. Flexible AC Transmission System (FACTS) device is an effective technology that has appeared as an alternative way in controlling power flow and damping power system oscillations. Although the damping ratio of FACTS-based stabilizer controllers is not often their main function, but its ability to increase power system damping characteristics has been acknowledged $[5,6]$.

Unified Power Flow Controller (UPFC) is one of the most important FACTS device families. Its primary function is to control and optimize the real and reactive power flow in a given line, voltage, and current at the UPFC bus [7, 8]. This is achieved by regulating the controllable parameters of the transmission system such as line impedance, phase angle, and voltage magnitude. Beside these primary functions, it can also provide appropriate damping effect to the tie interconnected modern power systems oscillation through its supplementary controller and increase system stability [9].

To improve general power system performance, possible interactions between PSSs and FACTS-damping controllers 


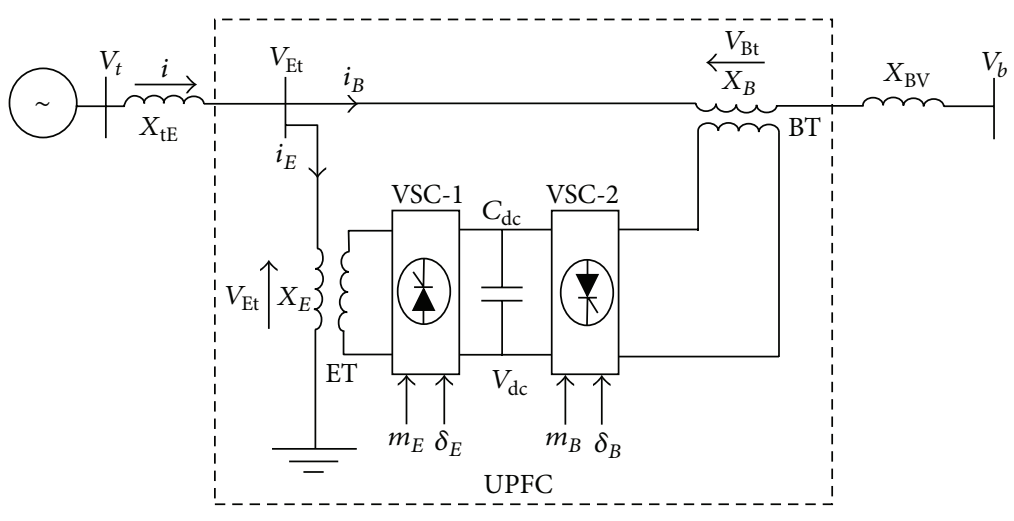

FIGURE 1: SMIB power system equipped with UPFC.

are considered, but uncoordinated local design of PSS and FACT-damping controller may cause destabilizing interaction on the damping of system oscillations [10]. Therefore, coordinated design of PSSs and FACTS-based stabilizers is a necessity both to make use of the benefits of multiple stabilizers, which will enhance the system stability and prevent the drawbacks accompanied with their operation.

Several researches have been carried out for the coordination between PSSs and FACTS damping controllers. Some of these researches were depending on the complex nonlinear simulation while the others based on the linearized model for power system [11-17]. One approach for achieving the required performance is to make the coordinated design of the controller as a constrained optimization problem. The constraints include limits of controller parameters. The most traditional optimization methods used some deterministic rules to move from one point to another in the decision hyperspace. Therefore, probability of getting stuck at a local optimum is the main problem with these methods [18].

Recently, Particle Swarm Optimization (PSO) technique has appeared as a useful tool for engineering global optimization. PSO is a population-based stochastic optimization method, which employs the swarm intelligence produced by the cooperation and rivalry between the particles in a swarm. PSO is unlike the other evolutionary algorithms having many advantages. The major advantages are as follows: it is fast and simple, does need to apply operators such as GA algorithm, and is easy to be implemented $[19,20]$. The simple PSO greatly depends on its parameters, and it is not guaranteed to be global convergent. In order to improve the global searching ability and prevent a slide into the premature convergence to local minima, PSO and chaotic sequence techniques are combined to form a Chaotic Particle Swarm Optimization (CPSO) technique.

In this paper, we present the results of our comprehensive comparison and assessment of the damping function of multiple damping stabilizers using different coordinated designs in order to identify the design that provided the most effective damping performance. The two alternative designs we evaluated are listed below: (i) coordinated design between PSS and any one out of the four input control channels of the series and shunt structure of UPFC device because any control loop can superimpose a supplementary damping controller to implement the required damping,

(ii) coordinated design between any two out of the four input control channels of the series and shunt structure of UPFC device as a multiple damping controller without using PSS.

The parameters of the damping controllers for individual and coordinated design are optimized utilizing CPSO technique based on eigenvalue objective function. The simulation results of the individual damping controllers show the best damping effects resulting from using the POD controllers $\delta_{E}$ and $m_{B}$ are higher than PSS response. In addition, the coordinated designs $\left(m_{B} \& \delta_{E}\right),\left(m_{E} \& \delta_{E}\right),\left(\delta_{E} \& \delta_{B}\right)$, and (PSS \& $\delta_{E}$ ) are able to damp the LFO effectively and can accomplish this task faster and with lower overshoot in comparison to their individual control responses or the best individual controls $\delta_{E}$ and $m_{B}$.

\section{Mathematical Model of Power System for SMIB Equipped with UPFC}

Figure 1 shows a single-machine infinite bus (SMIB) equipped with a UPFC. The synchronous generator is supplying electric power to the infinite bus through a transmission line and UPFC. The UPFC consists of two three-phase GTO based voltage source converters (VSCs) coupled through a common dc terminal. VSC-1 is connected in shunt with the line through an excitation transformer (ET), and VSC2 is inserted in series with the transmission line through a boosting transformer (BT). The dc voltage for both converters is provided by a common capacitor bank [21]. Among the four input control signals to the UPFC, $m_{E}$ and $\delta_{E}$ represent the amplitude modulation ratio and phase angle signals of shunt converter VSC-1; $m_{B}$ and $\delta_{B}$ represent the amplitude modulation ratio and phase angle signals of converter VSC2. These parameters are considered as UPFC control inputs to provide synchronized power compensation in series line without external voltage source $[4,22]$. 


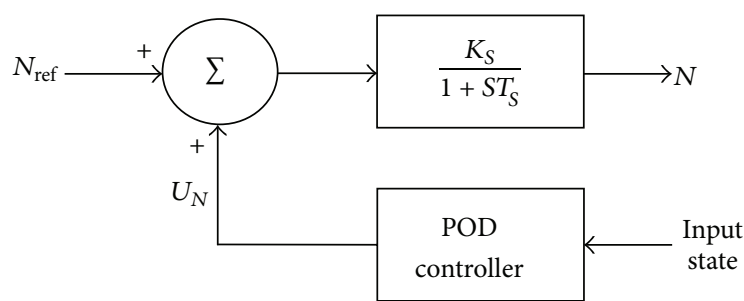

FIGURE 2: UPFC with damping controller.

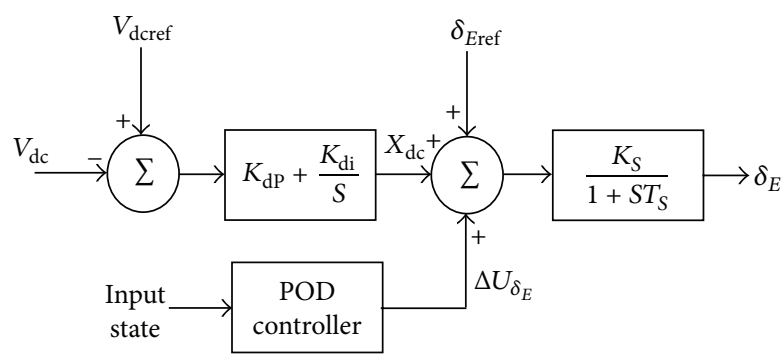

FIGURE 3: UFPC with dc voltage regulator and damping controller.

The structure of the UPFC controller is shown in Figure 2, where $N$ can be $m_{E}, m_{B}, \delta_{E}$, and $\delta_{B}$. In order to maintain the power flow between the series and shunt converters, a $\mathrm{dc}$ voltage regulator must be incorporated. The dc voltage is controlled through modulating the phase angle of the ET voltage $\delta_{E}$. Therefore, the $\delta_{E}$ damping controller is to be considered as shown in Figure 3, where the dc voltage regulator is a PI-controller [23].

2.1. Nonlinear Dynamic Model of UPFC. Referring to Figure 1, the nonlinear differential equations describe the dynamic behavior of the UPFC, the three-phase excitation current, three-phase boosting current, and dc link voltage which can be written as follows [24]:

$$
\begin{aligned}
{\left[\begin{array}{c}
\frac{d i_{E a}}{d t} \\
\frac{d i_{E b}}{d t} \\
\frac{d i_{E c}}{d t}
\end{array}\right]=} & {\left[\begin{array}{ccc}
\frac{-r_{E}}{l_{E}} & 0 & 0 \\
0 & \frac{-r_{E}}{l_{E}} & 0 \\
0 & 0 & \frac{-r_{E}}{l_{E}}
\end{array}\right]\left[\begin{array}{c}
i_{E a} \\
i_{E b} \\
i_{E c}
\end{array}\right] } \\
& -\frac{m_{E} v_{\mathrm{dc}}}{2 l_{E}}\left[\begin{array}{c}
\cos \left(\omega t+\delta_{E}\right) \\
\cos \left(\omega t+\delta_{E}-120^{\circ}\right) \\
\cos \left(\omega t+\delta_{E}+120^{\circ}\right)
\end{array}\right] \\
& +\left[\begin{array}{ccc}
\frac{1}{l_{E}} & 0 & 0 \\
0 & \frac{1}{l_{E}} & 0 \\
0 & 0 & \frac{1}{l_{E}}
\end{array}\right]\left[\begin{array}{l}
v_{E t a} \\
v_{E t b} \\
v_{E t c}
\end{array}\right],
\end{aligned}
$$

$$
\begin{aligned}
& {\left[\begin{array}{c}
\frac{d i_{B a}}{d t} \\
\frac{d i_{B b}}{d t} \\
\frac{d i_{B c}}{d t}
\end{array}\right]=\left[\begin{array}{ccc}
\frac{-r_{B}}{l_{B}} & 0 & 0 \\
0 & \frac{-r_{B}}{l_{B}} & 0 \\
0 & 0 & \frac{-r_{B}}{l_{B}}
\end{array}\right]\left[\begin{array}{c}
i_{B a} \\
i_{B b} \\
i_{B c}
\end{array}\right]} \\
& -\frac{m_{B} v_{\mathrm{dc}}}{2 l_{B}}\left[\begin{array}{c}
\cos \left(\omega t+\delta_{B}\right) \\
\cos \left(\omega t+\delta_{B}-120^{\circ}\right) \\
{[5 p t] \cos \left(\omega t+\delta_{B}+120^{\circ}\right)}
\end{array}\right] \\
& +\left[\begin{array}{ccc}
\frac{1}{l_{B}} & 0 & 0 \\
0 & \frac{1}{l_{B}} & 0 \\
0 & 0 & \frac{1}{l_{B}}
\end{array}\right]\left[\begin{array}{l}
v_{B t a} \\
v_{B t b} \\
v_{B t c}
\end{array}\right], \\
& \frac{d v_{\mathrm{dc}}}{d t}=\frac{m_{E}}{2 C_{\mathrm{dc}}}\left[\begin{array}{c}
\cos \left(\omega t+\delta_{E}\right) \\
\cos \left(\omega t+\delta_{E}-120^{\circ}\right) \\
\cos \left(\omega t+\delta_{E}+120^{\circ}\right)
\end{array}\right]^{T}\left[\begin{array}{c}
i_{E a} \\
i_{E b} \\
i_{E c}
\end{array}\right] \\
& +\frac{m_{B}}{2 C_{\mathrm{dc}}}\left[\begin{array}{c}
\cos \left(\omega t+\delta_{B}\right) \\
\cos \left(\omega t+\delta_{B}-120^{\circ}\right) \\
\cos \left(\omega t+\delta_{B}+120^{\circ}\right)
\end{array}\right]^{T}\left[\begin{array}{c}
i_{B a} \\
i_{B b} \\
i_{B c}
\end{array}\right],
\end{aligned}
$$

where $v_{E t}, i_{E}, r_{E}$, and $l_{E}$ are the excitation transformer voltage, current, resistance, and inductance, respectively; $v_{\mathrm{Bt}}, i_{B}, r_{B}$, and $l_{B}$ are the boosting voltage, current, resistance, and inductance respectively; $C_{\mathrm{dc}}, v_{\mathrm{dc}}$ are the dc link capacitance and voltage respectively.

By applying Park's transformation and ignoring the resistance and transient of the UPFC transformers, equations (1) become as follows [25]:

$$
\begin{aligned}
& {\left[\begin{array}{l}
v_{E t d} \\
v_{E t q}
\end{array}\right]=\left[\begin{array}{cc}
0 & -x_{E} \\
x_{E} & 0
\end{array}\right]\left[\begin{array}{l}
i_{E d} \\
i_{E q}
\end{array}\right]+\left[\begin{array}{l}
\frac{v_{\mathrm{dc}} m_{E} \cos \delta_{E}}{2} \\
\frac{v_{\mathrm{dc}} m_{E} \sin \delta_{E}}{2}
\end{array}\right],} \\
& {\left[\begin{array}{l}
v_{B t d} \\
v_{B t q}
\end{array}\right]=\left[\begin{array}{cc}
0 & -x_{B} \\
x_{B} & 0
\end{array}\right]\left[\begin{array}{l}
i_{B d} \\
i_{B q}
\end{array}\right]+\left[\begin{array}{l}
\frac{v_{\mathrm{dc}} m_{B} \cos \delta_{B}}{2} \\
\frac{v_{\mathrm{dc}} m_{B} \sin \delta_{B}}{2}
\end{array}\right] \text {, }} \\
& \frac{d v_{\mathrm{dc}}}{d t}=\frac{3 m_{E}}{4 C_{\mathrm{dc}}}\left[\begin{array}{ll}
\cos \delta_{E} & \sin \delta_{E}
\end{array}\right]\left[\begin{array}{l}
i_{E d} \\
i_{E q}
\end{array}\right] \\
& +\frac{3 m_{B}}{4 C_{\mathrm{dc}}}\left[\begin{array}{ll}
\cos \delta_{B} & \sin \delta_{B}
\end{array}\right]\left[\begin{array}{c}
i_{B d} \\
i_{B q}
\end{array}\right],
\end{aligned}
$$

where $x_{E}$ is the ET reactance and $x_{B}$ is the BT reactance. From 
Figure 1, we can have

$$
\begin{gathered}
\bar{V}_{t}=j x_{t E} \bar{I}+\bar{V}_{E t}, \\
\bar{V}_{E t}=\bar{V}_{B t}+j x_{B v} \bar{I}_{B}+\bar{V}_{b}, \\
\bar{I}=\bar{I}_{E}+\bar{I}_{B},
\end{gathered}
$$

where $I$ is the armature current, $V_{t}$ is the generator terminal voltage, $V_{b}$ is the infinite-bus voltage, $x_{t E}$ and $x_{B v}$ are the reactances of a transmission line. The terminal voltage and the armature current of the generator can be expressed in terms of the $d$-axis and $q$-axis components:

$$
\begin{gathered}
v_{t}=v_{t d}+j v_{t q} \\
v_{t d}=x_{q} i_{q} ; \quad v_{t q}=E_{q}^{\prime}-\grave{x}_{d} i_{d} \\
i=i_{d}+i_{q} \\
i_{d}=i_{E d}+i_{B d} ; \quad i_{q}=i_{E q}+i_{B q},
\end{gathered}
$$

where $\grave{x}_{d}$ is the $d$-axis transient reactance, $x_{q}$ is the $q$-axis reactance, and $E_{q}^{\prime}$ is the transient generator internal voltage. Substituting $\left(6^{\prime}\right)$ and $\left(7^{\prime}\right)$ into (6) gives

$$
v_{t d}+j v_{t q}=x_{q}\left(i_{E q}+i_{B q}\right)+j\left[E_{q}^{\prime}-\grave{x}_{d}\left(i_{E d}+i_{B d}\right)\right]
$$

Equation (4) can be expressed in the $d$ - $q$ axis reference frame as:

$$
\begin{gathered}
v_{t d}+j v_{t q}=j x_{t E}\left(i_{E d}+j i_{E q}+i_{B d}+j i_{B q}\right)+v_{E t d}+j v_{E t q} \\
v_{E t d}+j v_{E t q}= \\
v_{B t d}+j v_{B t q}+j x_{B v} i_{B d}-x_{B v} i_{B q} \\
+v_{b} \sin \delta+j v_{b} \cos \delta .
\end{gathered}
$$

From (2), (8), and (9), we have obtained the current injection equations of UPFC:

$$
\begin{gathered}
i_{E d}=\frac{x_{B B}}{x_{d \Sigma}} E_{q}^{\prime}-\frac{m_{E} \sin \delta_{E} v_{\mathrm{dc}} x_{B d}}{2 x_{d \Sigma}} \\
+\frac{x_{d E}}{x_{d \Sigma}}\left(V_{b} \cos \delta+\frac{m_{B} \sin \delta_{B} v_{\mathrm{dc}}}{2}\right), \\
i_{E q}=\frac{m_{E} \cos \delta_{E} v_{\mathrm{dc}} x_{B q}}{2 x_{q \Sigma}}-\frac{x_{q E}}{x_{q \Sigma}}\left(V_{b} \sin \delta+\frac{m_{B} \cos \delta_{B} v_{\mathrm{dc}}}{2}\right), \\
i_{B d}=\frac{x_{E}}{x_{d \Sigma}} E_{q}^{\prime}+\frac{m_{E} \sin \delta_{E} v_{\mathrm{dc}} x_{d E}}{2 x_{d \Sigma}} \\
-\frac{x_{d t}}{x_{d \Sigma}}\left(V_{b} \cos \delta+\frac{m_{B} \sin \delta_{B} v_{\mathrm{dc}}}{2}\right),
\end{gathered}
$$

$$
i_{B q}=-\frac{m_{E} \cos \delta_{E} v_{\mathrm{dc}} x_{q E}}{2 x_{q \Sigma}}+\frac{x_{q t}}{x_{q \Sigma}}\left(V_{b} \sin \delta+\frac{m_{B} \cos \delta_{B} v_{\mathrm{dc}}}{2}\right),
$$

where

$$
\begin{gathered}
x_{B B}=x_{B}+x_{B v}, \\
x_{d \Sigma}=\left(\grave{x}_{d}+x_{t E}+x_{E}\right)\left(x_{B}+x_{B v}\right)+x_{E}\left(\grave{x}_{d}+x_{t E}\right) \\
x_{B d}=x_{B}+x_{B v}+\grave{x}_{d}+x_{t E}, \\
x_{d E}=\grave{x}_{d}+x_{t E}, \quad x_{B q}=x_{B}+x_{B v}+x_{q}+x_{t E} \\
x_{q \Sigma}=\left(x_{q}+x_{t E}+x_{E}\right)\left(x_{B}+x_{B v}\right)+x_{E}\left(x_{q}+x_{t E}\right), \\
x_{d t}=\grave{x}_{d}+x_{t E}+x_{E} \\
x_{q t}=x_{q}+x_{t E}+x_{E}, \quad x_{q E}=x_{q}+x_{t E},
\end{gathered}
$$

where $x_{d}$ is the $d$-axis reactance.

2.2. Nonlinear Model of the Power System. The nonlinear dynamic equations of the SMIB system shown in Figure 1 are described by [26]:

$$
\begin{gathered}
\dot{\delta}=\omega_{b}(\omega-1), \\
\dot{\omega}=\frac{\left[P_{m}-P_{e}-D(\omega-1)\right]}{M}, \\
\dot{E}_{q}^{\prime}=\frac{\left[E_{f d}-\left(x_{d}-\grave{x}_{d}\right) i_{d}-E_{q}^{\prime}\right]}{T_{d o}^{\prime}},
\end{gathered}
$$

where $\omega_{b}$ is the synchronous speed, $\delta$ and $\omega$ are the angle and speed of the rotor, respectively, $P_{m}$ and $P_{e}$ are the input mechanical and output electrical powers of the generator, respectively, $M$ and $D$ are the machine inertia constant and damping coefficient, respectively, $E_{f d}$ is the generator field voltage, and $T_{d o}^{\prime}$ is the open-circuit field time constant.

The excitation system is represented by a first-order model (IEEE type-ST1) [27]:

$$
\dot{E}_{f d}=\frac{\left[K_{a}\left(V_{\mathrm{ref}}-v_{t}\right)-E_{f d}\right]}{T_{a}},
$$

where $K_{a}$ and $T_{a}$ are the gain and time constant of excitation system, respectively; $v_{\text {ref }}$ is the reference voltage. 
The output power of the generator can be expressed in terms of the $d$-axis and $q$-axis components of the armature current and terminal voltage as

$$
P_{e}=v_{t d} i_{d}+v_{t q} i_{q}
$$

2.3. Linearized Model of a Power System Equipped with UPFC. Linear dynamic model of the power system is obtained by linearizing the nonlinear equations (2)-(14) around nominal operating point. The linearized model of the power system as shown in Figure 1 is given as follows:

$$
\begin{gathered}
\Delta \dot{\delta}=\omega_{b} \Delta \omega, \\
\Delta \dot{\omega}=\frac{\left(\Delta P_{m}-\Delta P_{e}-D \Delta \omega\right)}{M}, \\
\Delta \dot{E}_{q}^{\prime}=\frac{\left[-\Delta E_{q}^{\prime}+\Delta E_{f d}-\left(x_{d}-\grave{x}_{d}\right) \Delta i_{d}\right]}{T_{d o}^{\prime}}, \\
\Delta \dot{E}_{f d}=\frac{\left(-K_{a} \Delta v_{t}-\Delta E_{f d}\right)}{T_{a}},
\end{gathered}
$$

where

$$
\begin{aligned}
\Delta P_{e}= & K_{1} \Delta \delta+K_{2} \Delta E_{q}^{\prime}+K_{p c} \Delta v_{\mathrm{dc}}+K_{p e} \Delta m_{E} \\
& +K_{p \delta e} \Delta \delta_{E}+K_{p b} \Delta m_{B}+K_{p \delta b} \Delta \delta_{B}, \\
\Delta E_{q}^{\prime}= & K_{4} \Delta \delta+K_{3} \Delta E_{q}^{\prime}+K_{q c} \Delta v_{\mathrm{dc}}+K_{q e} \Delta m_{E} \\
& +K_{q \delta e} \Delta \delta_{E}+K_{q b} \Delta m_{B}+K_{q \delta b} \Delta \delta_{B}, \\
\Delta v_{t}= & K_{5} \Delta \delta+K_{6} \Delta E_{q}^{\prime}+K_{v c} \Delta v_{\mathrm{dc}}+K_{v e} \Delta m_{E} \\
& +K_{v \delta e} \Delta \delta_{E}+K_{v b} \Delta m_{B}+K_{v \delta b} \Delta \delta_{B}, \\
\Delta \dot{v}_{\mathrm{dc}}= & K_{7} \Delta \delta+K_{8} \Delta E_{q}^{\prime}-K_{9} \Delta v_{\mathrm{dc}}+K_{c e} \Delta m_{E} \\
& +K_{c \delta e} \Delta \delta_{E}+K_{c b} \Delta m_{B}+K_{c \delta b} \Delta \delta_{B},
\end{aligned}
$$

where the linearization constants $K_{1}-K_{9}, K_{p c}, K_{p e}, K_{p \delta e}, K_{p b}$, $K_{p \delta b}, K_{q c}, K_{q e}, K_{q \delta e}, K_{q b}, K_{q \delta b}, K_{v c}, K_{v e}, K_{v \delta e}, K_{v b}, K_{v \delta b}, K_{c e}$, $K_{c \delta e}, K_{c b}$, and $K_{c \delta b}$ are functions of the system parameters and the initial operating conditions.
Referring to Figure 2, the UPFC dynamic models of $\Delta m_{E}$, $\Delta m_{B}$, and $\Delta \delta_{B}$ are described by the following state equations:

$$
\begin{aligned}
\Delta \dot{m}_{E} & =\frac{1}{T_{s 1}}\left(-\Delta m_{E}+K_{S 1} \Delta U_{m E}\right), \\
\Delta \dot{m}_{B} & =\frac{1}{T_{S 3}}\left(-\Delta m_{B}+K_{S 3} \Delta U_{m B}\right), \\
\Delta \dot{\delta}_{B} & =\frac{1}{T_{S 4}}\left(-\Delta \delta_{B}+K_{S 4} \Delta U_{\delta B}\right) .
\end{aligned}
$$

In addition, from Figure 3, the UPFC dynamic model of a dc voltage regulator is described by the following state equations:

$$
\begin{aligned}
\Delta & \dot{\delta}_{E}=\frac{1}{T_{S 2}}\left(K_{S 2} \Delta X_{\mathrm{dc}}-\Delta \delta_{E}+K_{S 2} \Delta U_{\delta E}\right) \\
\Delta \dot{X}_{\mathrm{dc}}= & -K_{d P} K_{7} \Delta \delta-K_{d P} K_{8} \Delta E_{q}^{\prime}-\left(K_{d i}+K_{d P} K_{9}\right) \Delta v_{\mathrm{dc}} \\
& -K_{d P} K_{c e} \Delta m_{E}-K_{d P} K_{c \delta e} \Delta \delta_{E} \\
& -K_{d P} K_{c b} \Delta m_{B}-K_{d P} K_{c \delta b} \Delta \delta_{B},
\end{aligned}
$$

where $K_{d P}$ and $K_{d i}$ are the proportional and integral gains of the dc voltage regulator controller, respectively; $\left(K_{S 1}-K_{S 4}\right)$ and $\left(T_{S 1}-T_{S 4}\right)$ are the gains and times constants of the main control loops for UPFC, respectively.

Equation (15) describes the model of the machine. Equations (16) and (18) represent the models for the exciter and the UPFC dc voltage regulators link, respectively. Equations (19) and (20) represent the control action of the main control loops of the UPFC with damping controller, and (21) represents the control action of PI dc voltage regulator. In state-space representation, these equations can be arranged in compact form as

$$
\Delta \dot{X}=A \Delta X+B \Delta U
$$

where the state vector $\Delta X$ and control vector $\Delta U$ are

$$
\begin{aligned}
& \Delta X=
\end{aligned}
$$

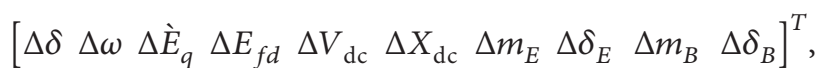

$$
\begin{aligned}
& \Delta U=\left[\begin{array}{llll}
\Delta U_{m E} & \Delta U_{\delta E} & \Delta U_{m B} & \Delta U_{\delta B}
\end{array}\right]^{T} .
\end{aligned}
$$

The structure of the matrices $A$ and $B$ is 


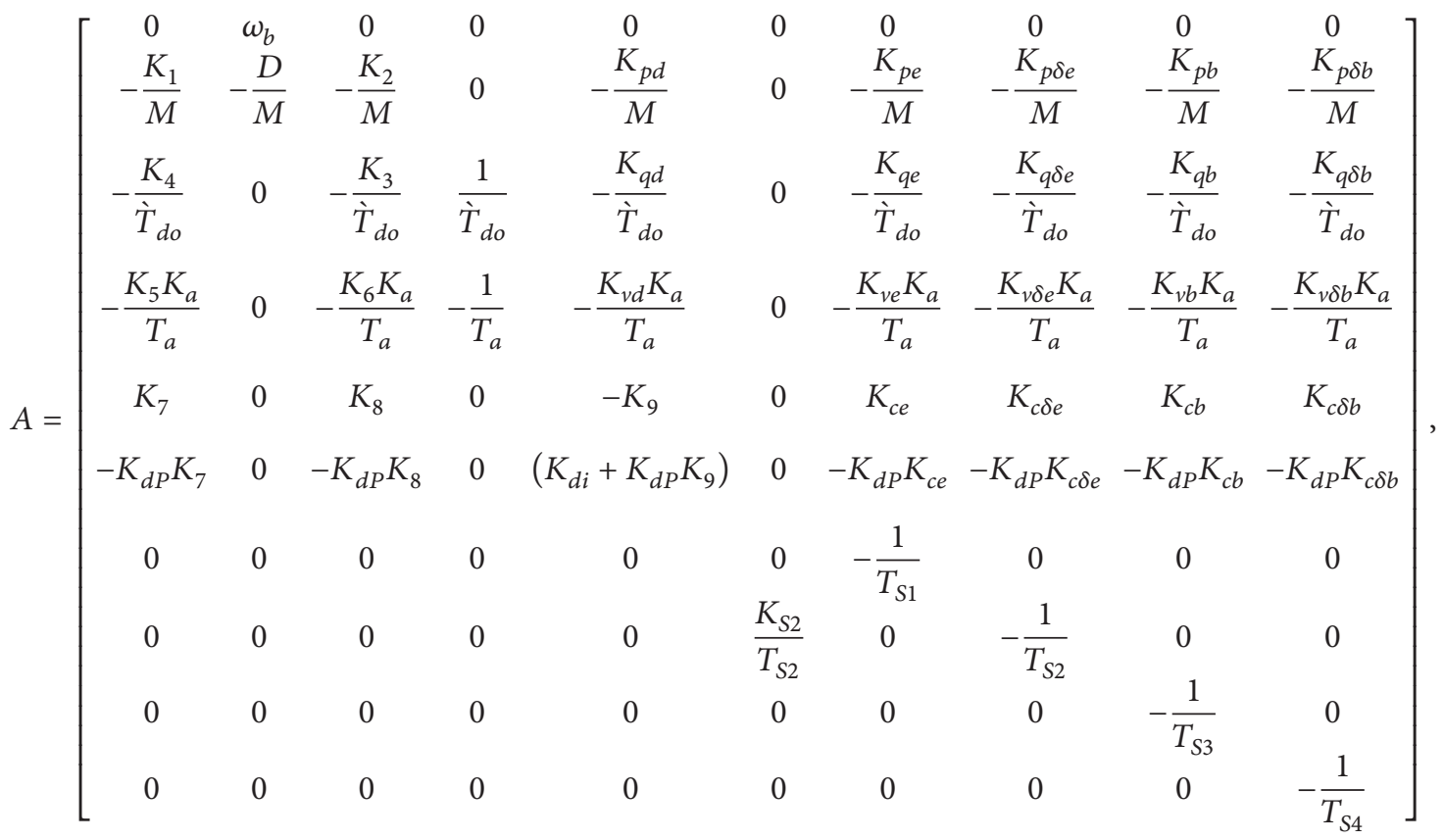

$$
\begin{aligned}
& B=\left[\begin{array}{cccc}
0 & 0 & 0 & 0 \\
0 & 0 & 0 & 0 \\
0 & 0 & 0 & 0 \\
0 & 0 & 0 & 0 \\
0 & 0 & 0 & 0 \\
0 & 0 & 0 & 0 \\
\frac{K_{S 1}}{T_{S 1}} & 0 & 0 & 0 \\
0 & \frac{K_{S 2}}{T_{S 2}} & 0 & 0 \\
0 & 0 & \frac{K_{S 3}}{T_{S 3}} & 0 \\
0 & 0 & 0 & \frac{K_{S 4}}{T_{S 4}}
\end{array}\right] .
\end{aligned}
$$

The modified Phillips-Heffron transfer function model including UPFC has 28 constants; on the other hand, the Phillips-Heffron model has only 6 constants as shown in Figure 4. This figure shows that the damping torque provided by the UPFC device consists of two parts. The first part is applied directly to the electromechanical oscillation loop of the generator. Its sensitivity is mainly measured by coefficients $K_{p c}, K_{p e}, K_{p \delta e}, K_{p b}$, and $K_{p \delta b}$, which is called the direct-damping torque. The second part consists of coefficients $K_{v c}, K_{v e}, K_{v \delta e}, K_{v b}$, and $K_{v \delta b}$, which is applied through the field channel of the generator. Its sensitivity is related to the deviation of field voltage, which is referred to as the indirect-damping torque.

2.4. Eigenvalue Analysis without Stabilizer. For nominal operating condition, the dynamic behavior of the system is recognized through the eigenvalues of the system matrix $A$.
By solving the system characteristic equation $|\lambda I-A|=0$, the eigenvalues of the system are computed which are given below:

$$
\begin{gathered}
\lambda_{1}=-19.752, \quad \lambda_{2}=-19.826, \\
\lambda_{3}=0.48394+3.4508 i, \quad \lambda_{4}=0.48394-3.4508 i, \\
\lambda_{5}=-1.7178, \quad \lambda_{6}=-0.13878+0.30433 i \\
\lambda_{7}=-0.13878-0.30433 i, \quad \lambda_{8}=-20 \\
\lambda_{9}=-20, \quad \lambda_{10}=-20 .
\end{gathered}
$$

It is clearly seen from eigenvalues of the matrix $A$ that the system is unstable and needs a supplementary stabilizer for stability. 


\section{Structure of the Supplementary Damping Stabilizers PSS and POD Controller}

In order to overcome the LFO problem, supplemental control action is applied to the generator excitation in the form of PSS or UPFC device as POD controller. The four main control parameters of the UPFC $\left(m_{E}, \delta_{E}, m_{B}\right.$, and $\left.\delta_{B}\right)$ can be modulated in order to produce the appropriate damping torque, which is illustrated in Figure 2.

The POD controller has a structure similar to that of the PSS. Figure 5 shows a sample block diagram of a POD controller. The controller contains three main blocks, that is, the gain block, the washout filter block, and two blocks (leadlag) phase compensators. It provides an electrical torque in phase with the speed deviation in order to improve damping of power system oscillations. The washout filter block acts as a high-pass filter to eliminate the dc offset of the POD output and prevent steady-state changes in the terminal voltage of the generator. From this perspective, the washout time $T_{\omega}$ should have a value in the range of 1 to 20 seconds defined to the electromechanical oscillation modes [1]. In this study, the time constants $T_{\omega}, T_{2}$, and $T_{4}$ were assigned specific values of $10 \mathrm{~s}, 0.1 \mathrm{~s}$, and $0.1 \mathrm{~s}$, respectively, while the parameters of the POD controller such as $K_{N}, T_{1}$, and $T_{3}$ need to be determined. The speed deviation $\Delta \omega$ used as an input signal to the POD and $\Delta U_{N}$ is the controller output, where $N=$ PSS, $m_{E}, \delta_{E}, m_{B}$, and $\delta_{B}$. With usage of the above dynamic damping controller, the number of matrix state variables increases from 10 to 13 , by adding three state variables $\Delta \dot{X}_{1}, \Delta \dot{X}_{2}$, and $\Delta \dot{U}_{N}$.

3.1. Optimal Design of the Supplementary Damping Stabilizers (PSS or POD). The main objective of optimization technique is to improve the dynamic stability of the power system against disturbances at different loading conditions. It can be achieved by suitable tuning of damping controller parameters.

The supplementary damping stabilizer (lead-lag type) can be described mathematically as

$$
U(s)=G(s) Y(s),
$$

where $G(s)$ is the transfer function of the supplementary damping stabilizer, $Y(s)$ is the measurement signal, and $U(s)$ is the output signal of the supplementary damping stabilizer, which will provide additional damping by moving electromechanical mode to the left. Equation (26) can be expressed in state-space form as

$$
\Delta \dot{X}_{C}=A_{C} \Delta X_{C}+B_{C} \Delta U
$$

where $\Delta X_{C}$ is the controller state vector. Equation (22) describes a linear model of the power system extracted around a certain operating point. combining (22) with (27), we obtained a closed-loop system:

$$
\begin{gathered}
\Delta \dot{X}_{C \ell}=A_{c \ell} \Delta X_{C \ell}, \\
\Delta X_{C \ell}=\left[\begin{array}{c}
\Delta X \\
\Delta X_{C}
\end{array}\right], \\
\zeta_{i}=-\frac{\operatorname{Real}\left(\lambda_{i}\right)}{\left|\lambda_{i}\right|}, \\
J=\min \left(\zeta_{i}\right),
\end{gathered}
$$

where $\Delta X_{C \ell}$ is the state vector of the closed loop system, $\lambda_{i}$ is the $i$ th eigenvalue mode of the closed loop matrix $A_{c \ell}$ and $\zeta_{i}$ is the damping coefficient of the $i$ th eigenvalue. It is clear that the objective function $J$ will identify the minimum value of the damping coefficient among all modes.

The goal of optimization process is to maximize $J$ in order to realize appropriate damping for all modes including electromechanical mode, by exploring the search space of admissible control parameters, which enhance the system damping characteristics, and maximum $J$ is searched within the limited range of control parameters:

$$
\begin{gathered}
K_{N}^{\min } \leq K_{N} \leq K_{N}^{\max }, \quad T_{N i}^{\min } \leq T_{N i} \leq T_{N i}^{\max }, \\
N=\text { PSS, } m_{E}, \delta_{E}, m_{B}, \delta_{B}, \quad i=1,3 .
\end{gathered}
$$

Typical ranges of the optimized parameters are 0.01-100 for $K_{N}$ and $0.001-1$ for $T_{N i}$.

3.2. Optimization Process. The problem of tuning the parameters for individual and coordinated design for multiple damping controllers, which would ensure maximum damping performance, was solved via a PSO optimization procedure that appeared to be a promising evolutionary technique for handling optimization problems. PSO is a populationbased, stochastic-optimization technique that was inspired by the social behavior of flocks of birds and schools of fish [28].

The advantages of PSO algorithm are that it is simple and easy to implement and it has a flexible and well-balanced mechanism to enhance the local and global exploration capabilities. Recently, it has acquired wide range of applications in solving optimization design problems featuring nonlinearity, nondifferentiability, and high dimensionality in many area search spaces $[29,30]$.

3.2.1. Classical PSO Algorithm. In the PSO, each possible solution is represented as a particle, and each set of particles comprises a population. Each particle keeps its position in hyperspace, which is related to the fittest solution it ever experiences in a special memory called pbest. In addition, the position related to the best value obtained so far by any particle in the population is called gbest. For each iteration of the PSO algorithm, the pbest and gbest values are updated 


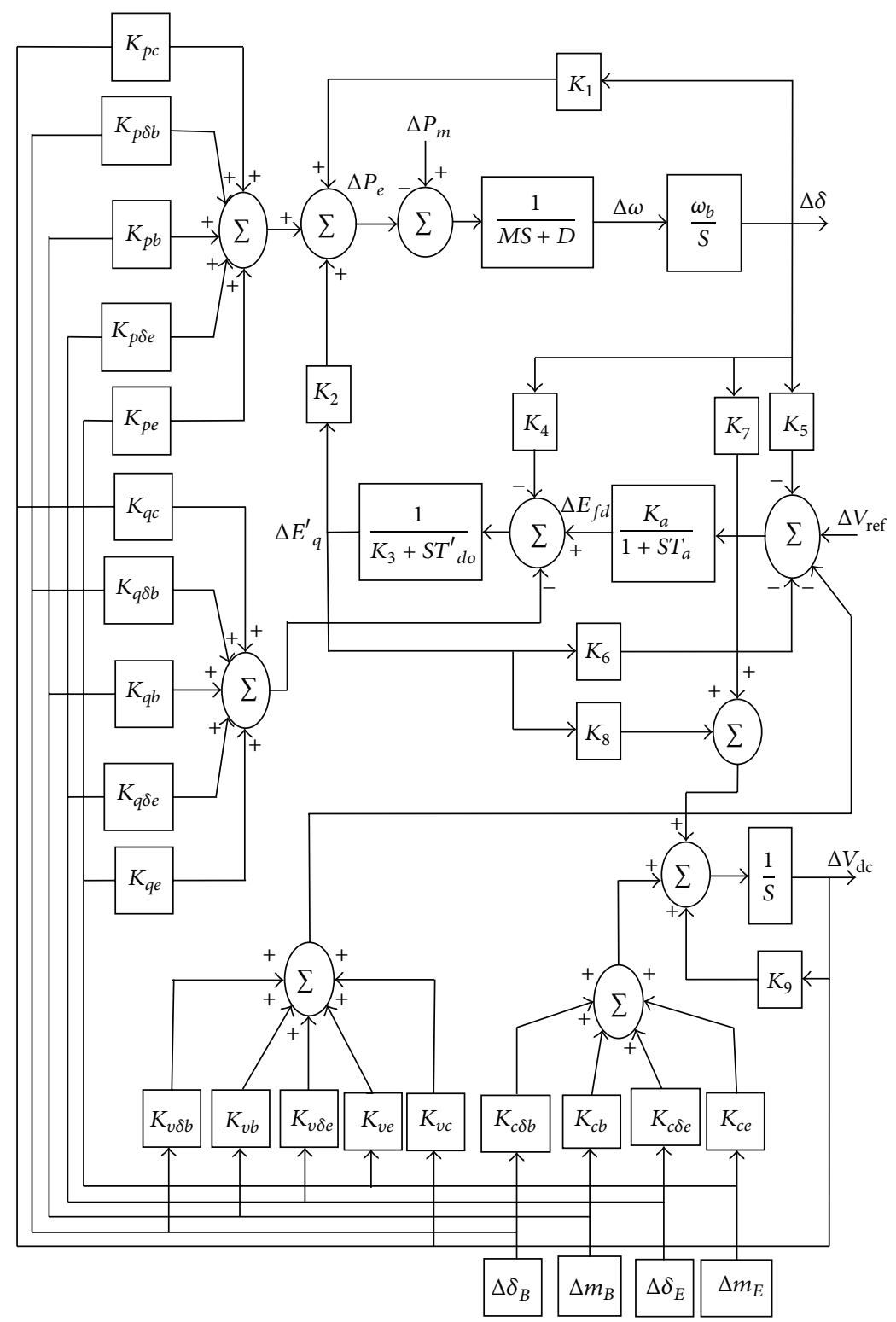

FIgURE 4: Modified Phillips-Heffron transfer function model of SMIB system with UPFC.

and each particle changes its velocity toward them randomly. This concept can be expressed as [18]

$$
\begin{gathered}
v_{i}^{k+1}=w v_{i}^{k}+c_{1} r_{1}\left(\text { pbest }_{i}-x_{i}^{k}\right)+c_{2} r_{2}\left(\text { gbest }-x_{i}^{k}\right) \\
x_{i}^{k+1}=x_{i}^{k}+v_{i}^{k+1}, \quad i=1,2, \ldots, n,
\end{gathered}
$$

where $v$ is the particle velocity, $x$ is the particle position, $k$ is the number of iterations, $w$ is the inertia weight factor, $c_{1}$ and $c_{2}$ are the cognitive and asocial acceleration factors respectively, $n$ is the number of particles, and $r_{1}$ and $r_{2}$ are the uniformly distributed random numbers in the range of 0 to 1 . Figure 6 shows the flow chart of the PSO algorithm.

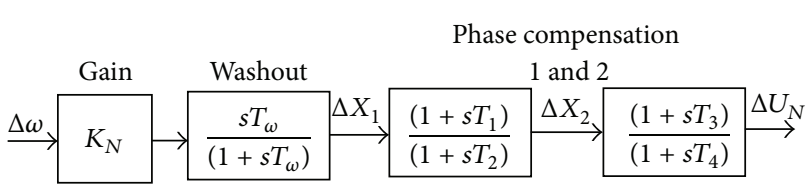

FIGURE 5: Structure of the supplementary damping stabilizers (PSS or POD).

3.2.2. Chaotic Particle Swarm Optimization (CPSO). The main disadvantage of the simple PSO algorithm is that the performance of it greatly depends on its parameters and it is not guaranteed to be global convergent. In order to improve the global searching ability and prevent a slide into 


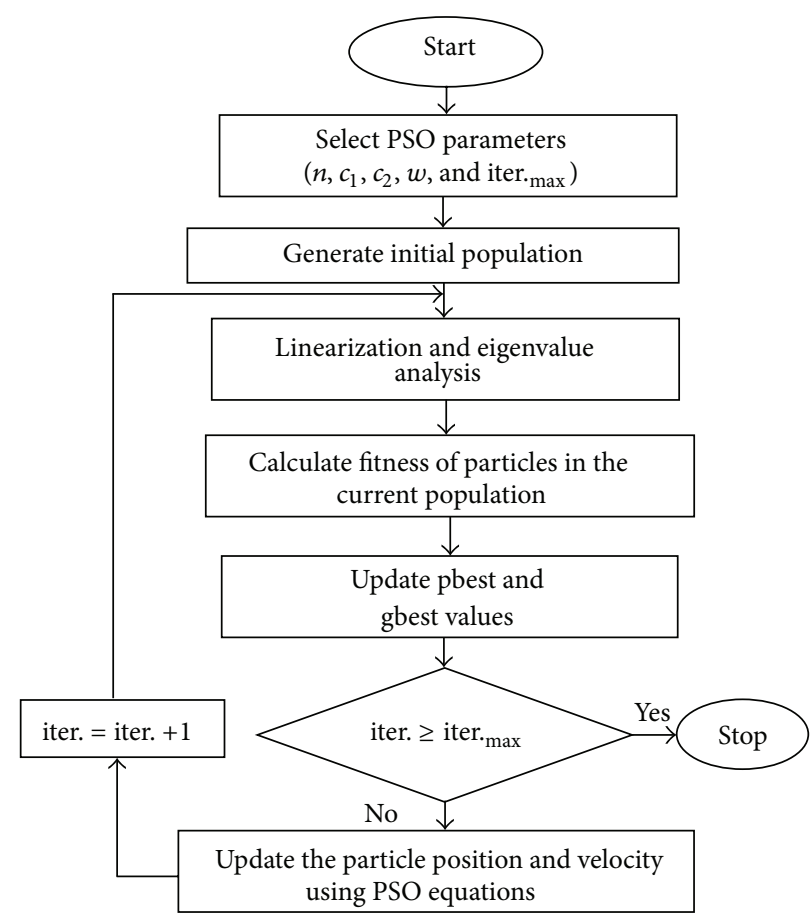

FIGURE 6: PSO algorithm for the tuning parameters of an individual and coordinated design.

the premature convergence to local minima, PSO and chaotic sequence techniques are combined to form a chaotic particle swarm optimization (CPSO) technique, which practically combines the population-based evolutionary searching ability of PSO and chaotic searching behavior. The logistic equation employed for constructing hybrid PSO is described as [31]:

$$
\beta^{k+1}=\mu \beta^{k}\left(1-\beta^{k}\right), \quad 0 \leq \beta^{1} \leq 1,
$$

where $\mu$ is the control parameter with a real value between 0 to 4. Although (32) is deterministic, it exhibits chaotic dynamics when $\mu=4$ and $\beta^{0} \notin\{0,0.25,0.5,0.75,1\}$. It exhibits the sensitive dependence on initial conditions, which is the basic characteristic of chaos. The inertia weighting function in (30) is usually evaluated utilizing the following equation:

$$
w=w_{\max }-\left[\left(w_{\max }-w_{\min }\right)\left(\frac{\text { iter }}{\text { iter }_{\max }}\right)\right],
$$

where $w_{\max }$ and $w_{\min }$ are maximum and minimum values of $w$, iter $_{\max }$ is the maximum number of iterations, and iter is the current iteration number. The new weight parameter $w_{\text {new }}$ is defined by multiplying weight parameter $w$ in (33) and logistic equation (32):

$$
w_{\text {new }}=w \times \beta^{k+1} .
$$

To improve the global searching capability of PSO, we have to introduce a new velocity update equation as follows:

$$
v_{i}^{k+1}=w_{\text {new }} v_{i}^{k}+c_{1} r_{1}\left(\text { pbest }_{i}-x_{i}^{k}\right)+c_{2} r_{2}\left(\text { gbest }-x_{i}^{k}\right) .
$$

We have observed that the proposed new weight decreases and oscillates simultaneously for total iteration, whereas the conventional weight decreases monotonously from $w_{\max }$ to $w_{\min }$. The final choice of a parameter was considered to be the optimal choice: $n$, iter ${ }_{\max }, c_{1}, c_{2}, w_{\min }$, $w_{\max }, \mu$, and $\beta^{0}$ are chosen as $30,100,2,2,0.3,0.9,4$, and 0.3 , respectively.

\section{Simulation and Comparison Results}

In this section, the ability of UPFC in damping system oscillation and the dynamic interactions of UPFC-POD controllers are investigated intensively. The CPSO technique has been applied to design individual and coordinated damping controllers. To evaluate the performance of the proposed simultaneous coordinated design approaches, the responses with the proposed controllers were compared with the responses of the individual design of the PSS and UPFCPOD controllers for two schemes. The resultant optimal parameters of the individual controllers and coordinated designs are given in Tables 1, 2, and 3, respectively.

4.1. Scheme 1. Figures 7-11 show the effect of applying the individual controllers and coordinated designs for PSS and different UPFC-POD controllers in a SMIB of speed deviation of $\Delta \omega$ with $10 \%$ step change in mechanical input power. The system eigenvalues with the proposed individual stabilizers and coordinated designs for nominal operating condition are given in Tables 4 and 5, respectively. The first 
TABLE 1: The optimal parameters of the individual controllers.

\begin{tabular}{|c|c|c|c|c|}
\hline \multirow{2}{*}{ Individual controllers } & \multirow{2}{*}{ Type of algorithm } & \multicolumn{3}{|c|}{ Optimal values } \\
\hline & & K & $T_{1}$ (Sec.) & $T_{3}$ (Sec.) \\
\hline \multirow{2}{*}{ PSS } & CPSO & 38.8433 & 0.2490 & 0.2490 \\
\hline & PSO & 12.2151 & 0.8227 & 0.8227 \\
\hline \multirow{2}{*}{$m_{E}$} & CPSO & 26.1560 & 0.8812 & 0.0015 \\
\hline & PSO & 31.5718 & 0.3635 & 0.0344 \\
\hline \multirow{2}{*}{$\delta_{E}$} & CPSO & 11.5577 & 0.1960 & 0.0331 \\
\hline & PSO & 12.1740 & 0.2143 & 0.0028 \\
\hline \multirow{2}{*}{$m_{B}$} & CPSO & 66.2928 & 0.0011 & 0.2369 \\
\hline & PSO & 75.4243 & 0.0998 & 0.3171 \\
\hline \multirow{2}{*}{$\delta_{B}$} & CPSO & 99.6244 & 0.7206 & 0.0017 \\
\hline & PSO & 25.4779 & 0.0307 & 0.5386 \\
\hline
\end{tabular}

TABLE 2: The optimal parameters of the coordinated designs between PSS and UPFC-POD controllers.

\begin{tabular}{|c|c|c|c|c|c|c|c|}
\hline \multirow{2}{*}{ Coordinated designs } & \multirow{2}{*}{ Type of algorithm } & \multicolumn{6}{|c|}{ Optimal values } \\
\hline & & $K^{*}$ & $T_{1}^{*}(\mathrm{Sec})$. & $T_{3}^{*}$ (Sec.) & $K^{\#}$ & $T_{1}^{\#}$ (Sec.) & $T_{3}^{\#}$ (Sec.) \\
\hline \multirow{2}{*}{ PSS $^{*} \& m_{E}^{\#}$} & CPSO & 45.5204 & 0.0418 & 0.2659 & 38.3455 & 0.0316 & 0.6943 \\
\hline & PSO & 44.0127 & 0.2628 & 0.0613 & 52.8507 & 0.5057 & 0.0072 \\
\hline \multirow{2}{*}{ PSS $^{*} \& \delta_{E}^{\#}$} & CPSO & 8.1683 & 0.2501 & 0.1518 & 10.0208 & 0.1993 & 0.0361 \\
\hline & PSO & 5.2118 & 0.7275 & 0.0301 & 10.9942 & 0.1579 & 0.0284 \\
\hline \multirow{2}{*}{ PSS $^{*} \& m_{B}^{\#}$} & CPSO & 57.2645 & 0.1963 & 0.1756 & 54.6790 & 0.1057 & 0.2037 \\
\hline & PSO & 61.6657 & 0.2949 & 0.4783 & 59.6813 & 0.3536 & 0.0816 \\
\hline \multirow{2}{*}{ PSS $^{*} \& \delta_{B}^{\#}$} & CPSO & 63.0262 & 0.2377 & 0.2371 & 3.9384 & 0.7395 & 0.9415 \\
\hline & PSO & 15.2002 & 0.9788 & 0.0074 & 0.3729 & 0.5007 & 0.5611 \\
\hline
\end{tabular}

TABLE 3: The optimal parameters of the coordinated designs between different UPFC-POD controllers.

\begin{tabular}{|c|c|c|c|c|c|c|c|}
\hline \multirow{2}{*}{ Coordinated designs } & \multirow{2}{*}{ Type of algorithm } & \multicolumn{6}{|c|}{ Optimal values } \\
\hline & & $K^{*}$ & $T_{1}^{*}($ Sec. $)$ & $T_{3}^{*}(\mathrm{Sec})$. & $K^{\#}$ & $T_{1}^{\#}$ (Sec.) & $T_{3}^{\#}(\mathrm{Sec})$. \\
\hline \multirow{2}{*}{$m_{E}^{*} \& \delta_{E}^{\#}$} & CPSO & 50.7479 & 0.6966 & 0.0656 & 8.0139 & 0.0513 & 0.0530 \\
\hline & PSO & 57.7561 & 0.4707 & 0.0895 & 7.9823 & 0.0460 & 0.0136 \\
\hline \multirow{2}{*}{$m_{B}^{*} \& \delta_{B}^{\#}$} & CPSO & 97.9661 & 0.0014 & 0.0019 & 69.2724 & 0.0022 & 0.1816 \\
\hline & PSO & 68.0064 & 0.2596 & 0.0413 & 42.4356 & 0.2982 & 0.0070 \\
\hline \multirow{2}{*}{$m_{E}^{*} \& m_{B}^{\#}$} & CPSO & 26.1813 & 0.0081 & 0.8276 & 2.3838 & 0.6509 & 0.4527 \\
\hline & PSO & 41.5012 & 0.9941 & 0.0023 & 0.1583 & 0.8581 & 0.0941 \\
\hline \multirow{2}{*}{$\delta_{E}^{*} \& \delta_{B}^{\#}$} & CPSO & 89.0042 & 0.0735 & 0.0087 & 63.4526 & 0.0490 & 0.2352 \\
\hline & PSO & 4.7214 & 1.0000 & 0.6398 & 11.4511 & 0.1435 & 0.7305 \\
\hline \multirow{2}{*}{$m_{E}^{*} \& \delta_{B}^{\#}$} & CPSO & 99.9911 & 0.2171 & 0.0407 & 81.5964 & 0.0005 & 0.0063 \\
\hline & PSO & 17.2632 & 0.1916 & 0.8990 & 87.1383 & 0.1447 & 0.0056 \\
\hline \multirow{2}{*}{$m_{B}^{*} \& \delta_{E}^{\#}$} & CPSO & 57.8685 & 0.0530 & 0.2428 & 10.5448 & 0.0462 & 0.0788 \\
\hline & PSO & 65.7520 & 0.1876 & 0.0403 & 10.7924 & 0.0619 & 0.0561 \\
\hline
\end{tabular}

and second rows represent the electromechanical mode and their damping ratio $\zeta_{\mathrm{EM}}$ using participation factor to identify the eigenvalue associated with electromechanical mode. It is clear that the proposed controllers stabilize the system effectively. The maximum overshoot as well as the settling time of the system responses has been compared to the 


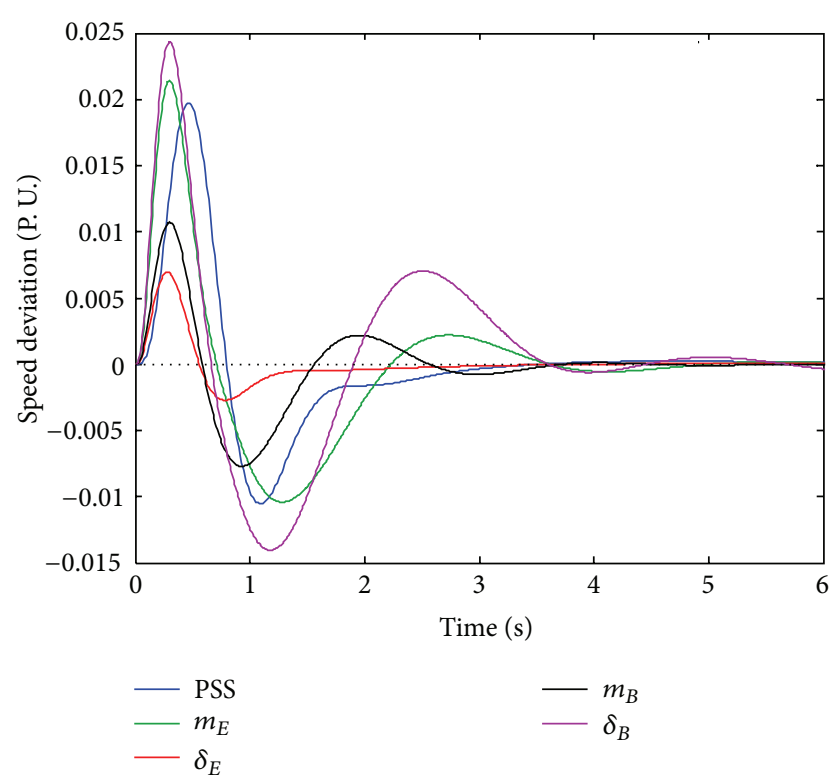

FIGURE 7: Speed variation responses for individual damping controllers (PSS, $m_{E}, \delta_{E}, m_{B}$, and $\delta_{B}$ ).

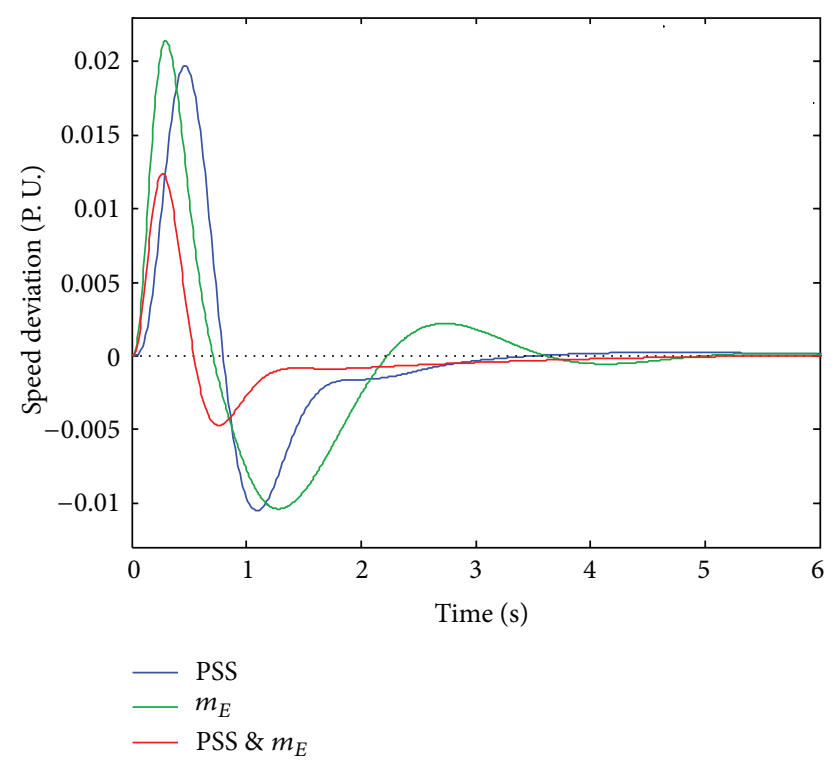

FIGURE 8: Speed variation responses for individual damping controllers PSS, $m_{E}$ and coordinated design PSS \& $m_{E}$.

capability of the simulated coordinate approaches in oscillation damping. Figure 7 shows the speed deviation responses of the four UPFC-POD controllers and PSS individually. All these responses are shown in one figure for comparison purposes. It can be seen that by using the UPFC-POD controllers $\delta_{E}$ and $m_{B}$ provide a robust damping characteristic higher than that of PSS and enhance the stability. However, the UPFC-POD controllers $m_{E}$ and $\delta_{B}$ do not perform well and have poor capabilities in damping the system oscillation.

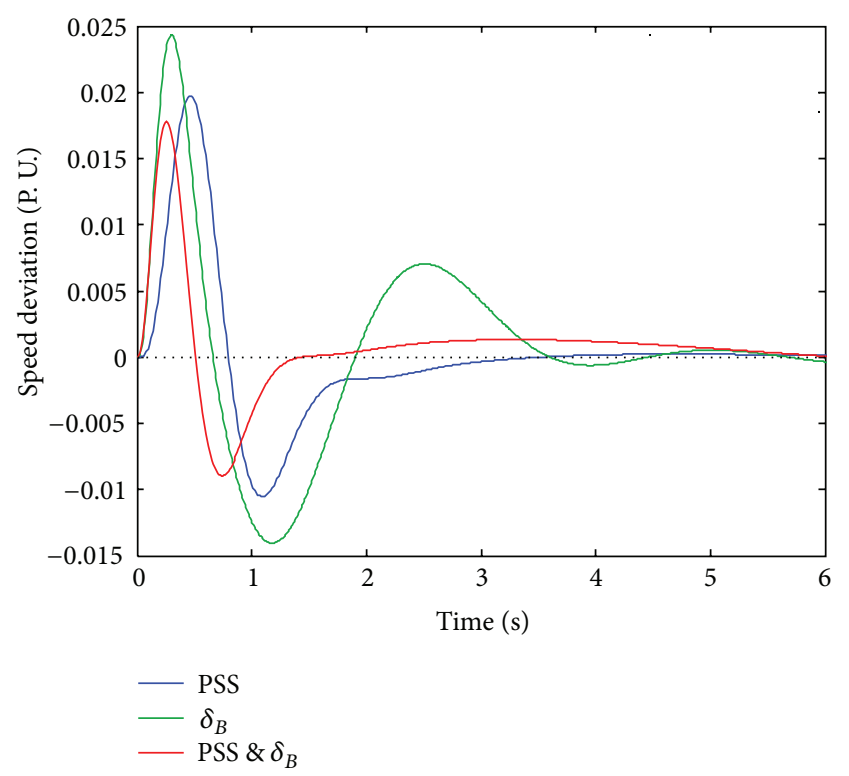

FIGURE 9: Speed variation responses for individual damping controllers PSS, $\delta_{B}$ and coordinated design PSS $\& \delta_{B}$.

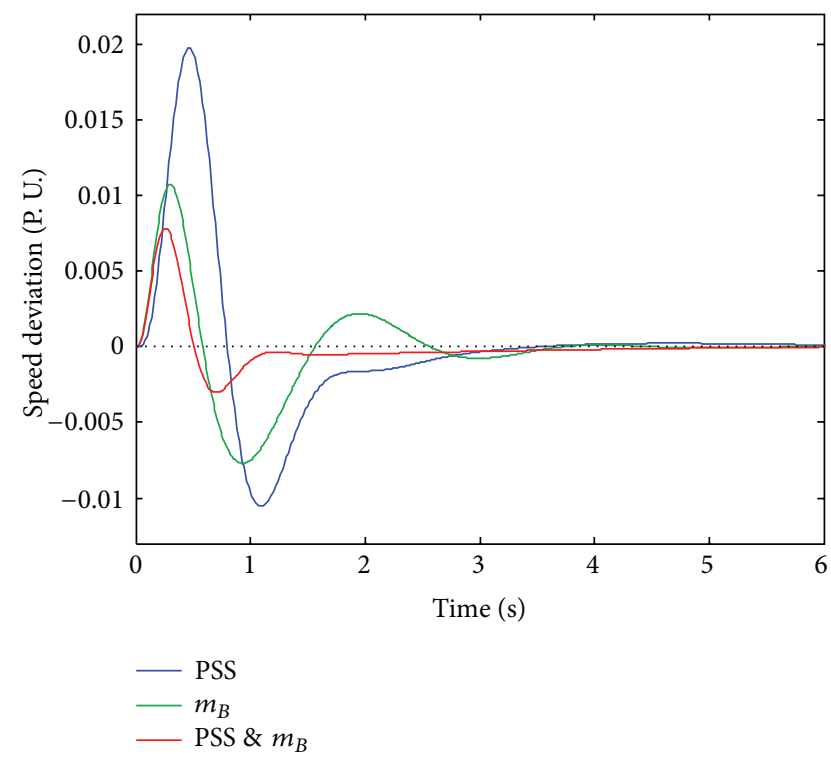

FIGURE 10: Speed variation responses for individual damping controllers PSS, $m_{B}$ and coordinated design PSS \& $m_{B}$.

Figures 8 and 9 show the improvement in overshoot and settling time of the system response while using the coordinated designs (PSS \& $m_{E}$ ) and (PSS \& $\delta_{B}$ ) over the individual control responses but less than the best individual controls $\delta_{E}$ and $m_{B}$. Figure 10 shows that the coordinated design (PSS \& $m_{B}$ ) improves the system damping compared to their individual controls but no more than the best one $\delta_{E}$. Figure 11 shows the best response in Scheme 1 while using the coordinated design (PSS \& $\delta_{E}$ ) over their individual control 


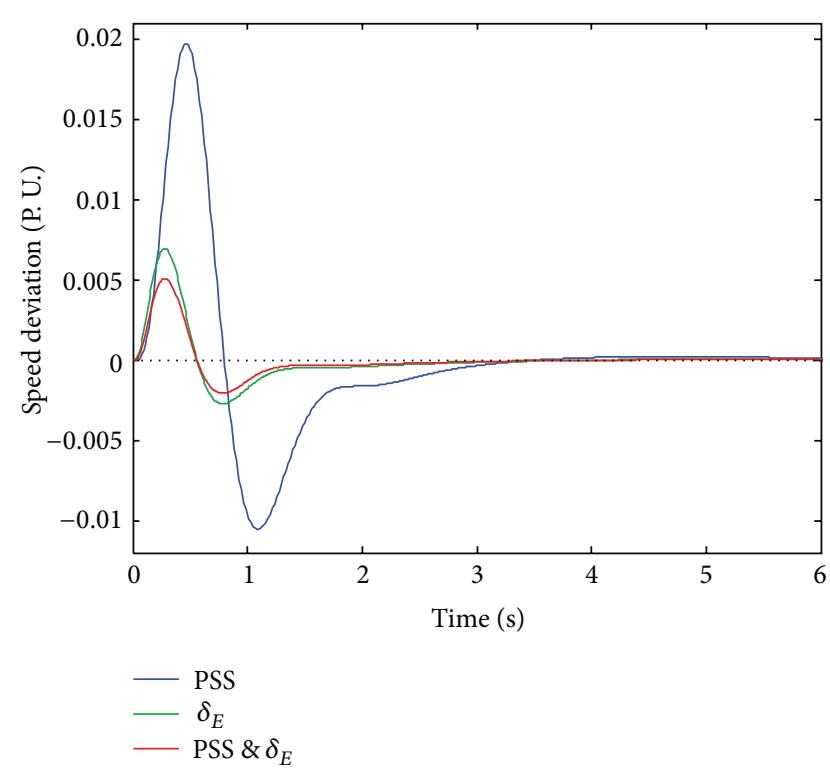

FIGURE 11: Speed variation responses for individual damping controllers PSS, $\delta_{E}$ and coordinated design PSS \& $\delta_{E}$.

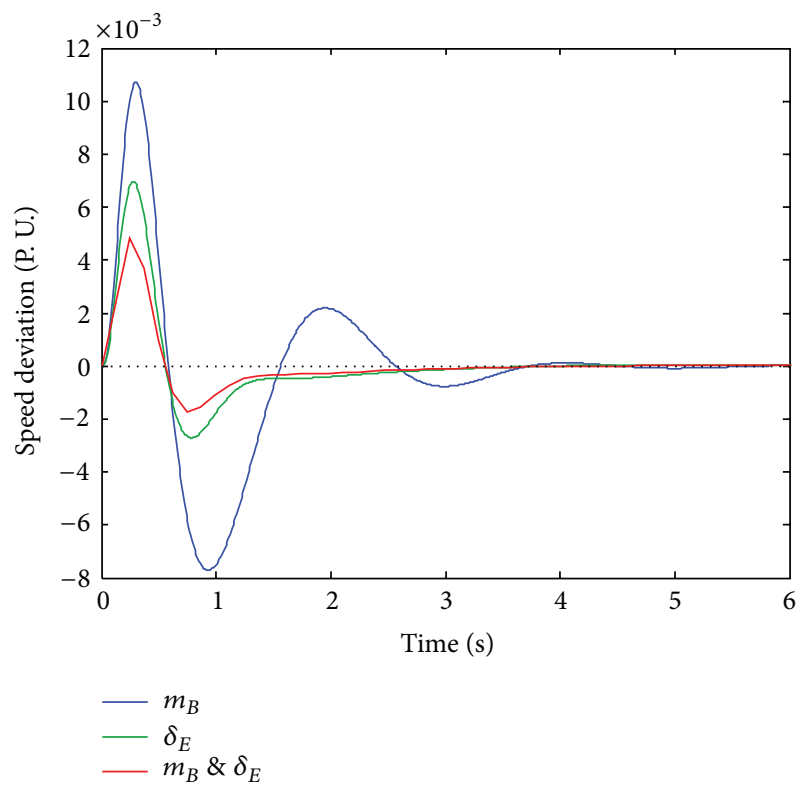

FIGURE 12: Speed variation responses for individual damping controllers $m_{B}, \delta_{E}$ and coordinated design $m_{B} \& \delta_{E}$.

responses because it improved both damping parameters overshoot and settling time.

4.2. Scheme 2. In this scheme, the power system is considered to possess a UPFC device without PSS. Figures 12-17 show the effect of applying the individual controllers and coordinated designs between different UPFC-POD controllers in a SMIB of speed deviation of $\Delta \omega$ with $10 \%$ step change in mechanical input power. The eigenvalues of the system with the proposed controllers in scheme 2 are given in Table 6.

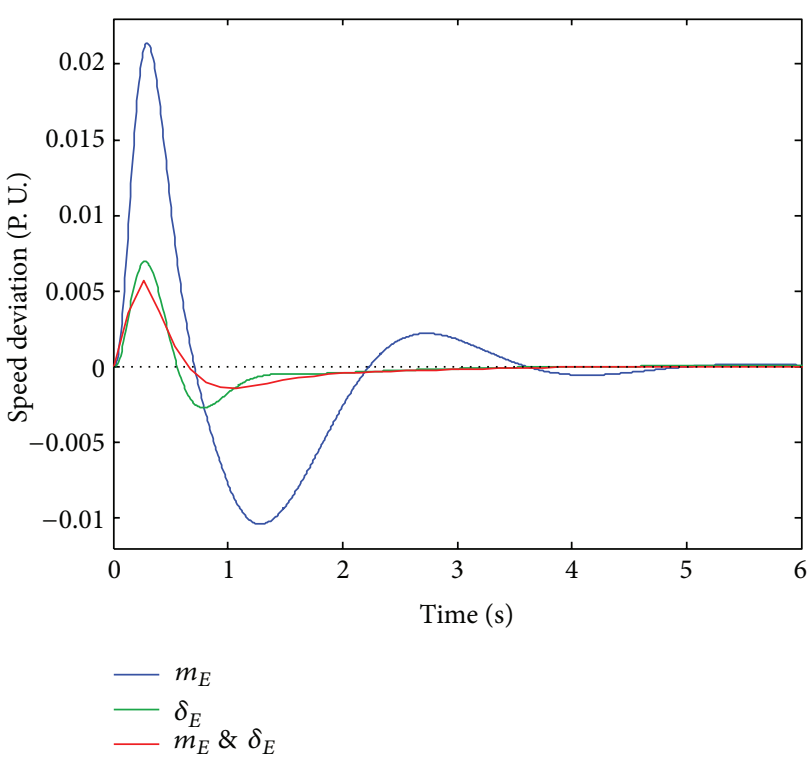

FIGURE 13: Speed variation responses for individual damping controllers $m_{E}, \delta_{E}$ and coordinated design $m_{E} \& \delta_{E}$.

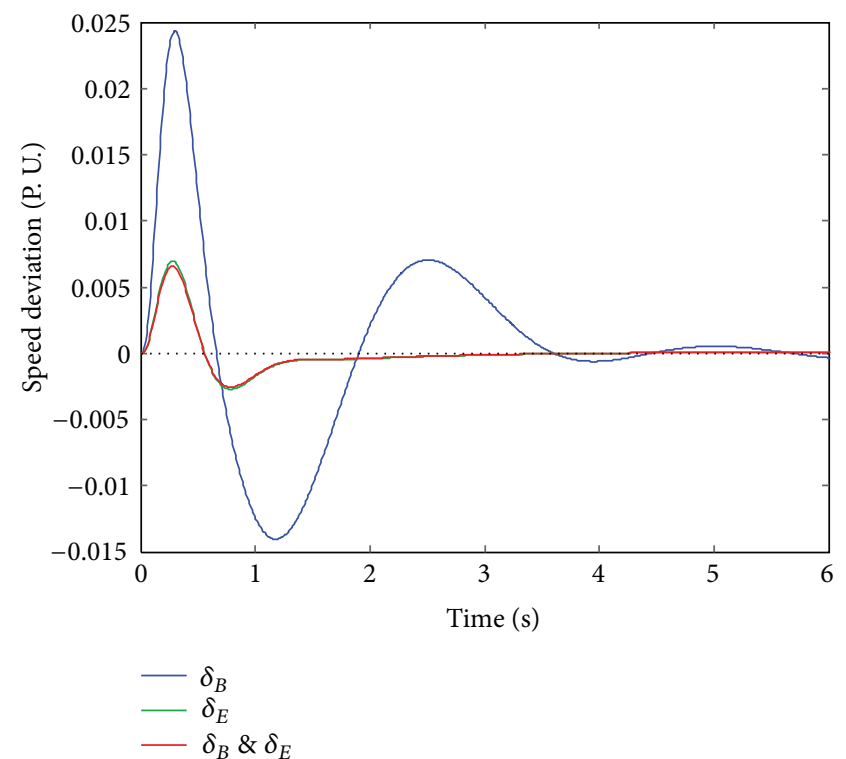

FIgURE 14: Speed variation responses for individual damping controllers $\delta_{B}, \delta_{E}$ and coordinated design $\delta_{B} \& \delta_{E}$.

It is clear that the proposed controllers stabilize the system effectively.

From Figures 12, 13, and 14, it is clear that the system damping resulting from using coordinated designs $\left(m_{B} \& \delta_{E}\right),\left(m_{E} \& \delta_{E}\right)$, and $\left(\delta_{E} \& \delta_{B}\right)$ are much higher than those resulting from using the best individual controls $\delta_{E}$ and $m_{B}$. Figures 15 and 16 show that the coordinated designs $\left(m_{E} \& m_{B}\right)$ and $\left(m_{B} \& \delta_{B}\right)$ improve the system damping compared to their individual controls but not more than the best one $\delta_{E}$. Figure 17 shows the improvement in overshoot 
TABle 4: System eigenvalues of the individual controllers.

\begin{tabular}{lcccc}
\hline PSS & $m_{E}$ & $\delta_{E}$ & $m_{B}$ & $\delta_{B}$ \\
\hline$-\mathbf{2 . 2 9} \pm \mathbf{j 7 . 0 2}$ & $-\mathbf{2 . 1 1} \pm \mathbf{j 7 . 3 1}$ & $-\mathbf{3 . 6 9} \pm \mathbf{j 6 . 0 8}$ & $-\mathbf{2 . 7 9} \pm \mathbf{j 5 . 1 1}$ & $\mathbf{- 1 . 3 8} \pm \mathbf{j 6 . 0 6}$ \\
$\boldsymbol{C}_{\mathrm{EM}}=\mathbf{0 . 3 1 0 1}$ & $\boldsymbol{\zeta}_{\mathrm{EM}}=\mathbf{0 . 2 7 7 3}$ & $\boldsymbol{\zeta}_{\mathrm{EM}}=\mathbf{0 . 5 1 8 8}$ & $\boldsymbol{\zeta}_{\mathrm{EM}}=\mathbf{0 . 4 7 9 2}$ & $\boldsymbol{\zeta}_{\mathrm{EM}}=\mathbf{0 . 2 2 2 0}$ \\
$-4.18 \pm j 8.27$ & $-5.38 \pm j 10$ & $-3.7 \pm j 5.56$ & $-2.24 \pm j 3.13$ & $-1.7 \pm j 4.25$ \\
$-3.78 \pm j 5.55$ & $-3.3 \pm j 7.22$ & $-4.38 \pm j 5.07$ & $-5.24 \pm j 7.46$ & $-2.09 \pm j 4.95$ \\
-24.84 & -28.96 & $-4.85 \pm 6.37$ & -25.27 & -28.52 \\
-19.83 & -16.58 & -23.72 & 19.17 & -19.71 \\
-0.69 & -19.87 & -19.83 & -1.93 & -16.49 \\
-0.33 & -2.14 & -0.26 & -0.2 & -1.98 \\
-20 & -0.21 & -20 & -20 & -0.29 \\
-20 & -20 & -20 & -20 & -20 \\
-20 & -20 & & -20 & -20 \\
\hline
\end{tabular}

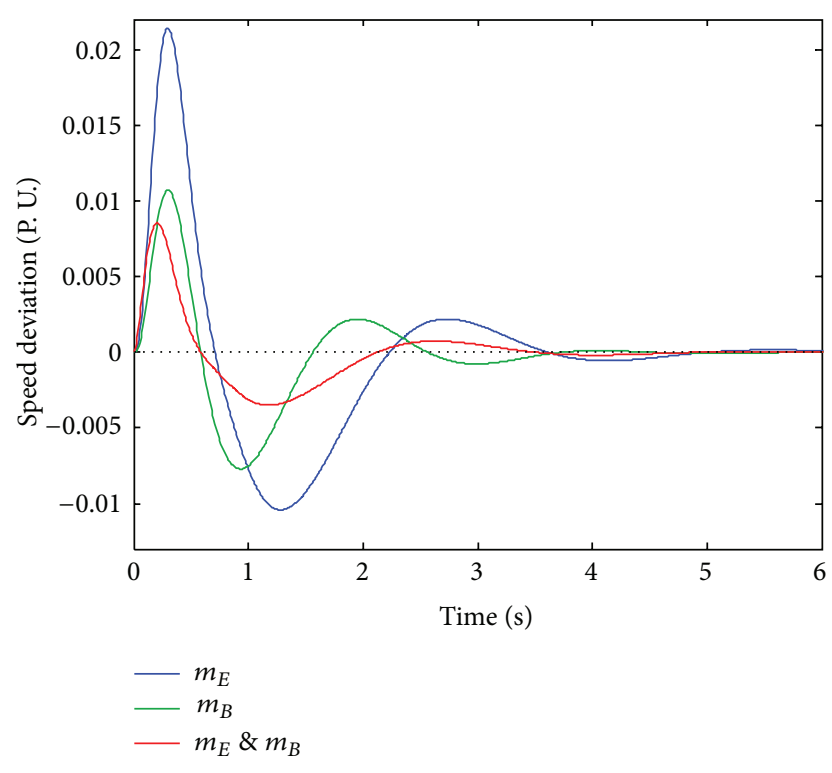

FIGURE 15: Speed variation responses for individual damping controllers $m_{E}, m_{B}$ and coordinated design $m_{E} \& m_{B}$.

and settling time of the system response while using the coordinated design $\left(m_{E} \& \delta_{B}\right)$ over the individual control responses but less than the best individual controls $\delta_{E}$ and $m_{B}$.

\section{Conclusions}

This paper is concerned with the damping of LFO via PSS and UPFC-POD controllers applied independently and also through the simultaneous coordinated designs in a SMIB power system. To improve the global searching ability and prevent a slide into the premature convergence to local minima, PSO and chaos theory are combined to form a CPSO. For the proposed controller design problem, a CPSO

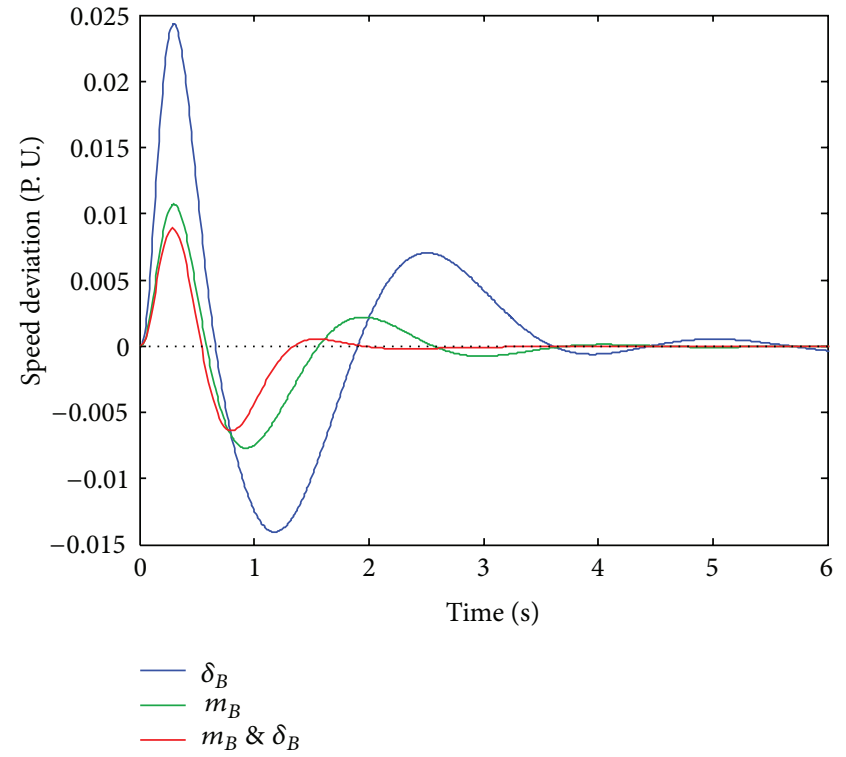

FIGURE 16: Speed variation responses for individual damping controllers $\delta_{B}, m_{B}$ and coordinated design $m_{B} \& \delta_{B}$.

algorithm was used as the optimization technique to search for the optimal damping controller parameters in both the individual and the coordinated designs. The simulation results of the individual damping controllers showed the best damping effects resulting from using $\delta_{E}$ and $m_{B}$ as POD controllers are much higher than those resulting from using PSS, $m_{E}$, and $\delta_{B}$ responses. In addition, the coordinated designs (PSS $\left.\& \delta_{E}\right)$ in scheme $1 ;\left(m_{B} \& \delta_{E}\right),\left(m_{E} \& \delta_{E}\right)$ and $\left(\delta_{E} \& \delta_{B}\right)$ in scheme 2 provide superior performance in comparison with the best individual controls $\delta_{E}$ and $m_{B}$ while the coordinated designs (PSS \& $\left.m_{B}\right)$ in scheme $1 ;\left(m_{E} \& m_{B}\right)$, and $\left(m_{B} \& \delta_{B}\right)$ in scheme 2 improve the system damping compared to the individual controls except $\delta_{E}$, which is 
TABLE 5: System eigenvalues of the coordinated designs between PSS and UPFC-POD controllers.

\begin{tabular}{lccr}
\hline PSS \& $m_{E}$ & PSS \& $\delta_{E}$ & PSS \& $m_{B}$ & \multicolumn{1}{c}{ PSS \& $\delta_{B}$} \\
\hline$-\mathbf{2 . 8 5} \pm \mathbf{j 5 . 6 7}$ & $-\mathbf{3 . 8 6} \pm \mathbf{j 5 . 3 2}$ & $-\mathbf{1 . 2 3} \pm \mathbf{j 2 . 1 1}$ & $-\mathbf{2 . 6 7} \pm \mathbf{j 6 . 3 9}$ \\
$\boldsymbol{\zeta}_{\mathrm{EM}}=\mathbf{0 . 4 5}$ & $\boldsymbol{\zeta}_{\mathrm{EM}}=\mathbf{0 . 5 8 7 3}$ & $\boldsymbol{\zeta}_{\mathrm{EM}}=\mathbf{0 . 5 0 4}$ & $\boldsymbol{\zeta}_{\mathrm{EM}}=\mathbf{0 . 3 8 5 5}$ \\
$-6.03 \pm j 6.11$ & $-4.94 \pm j 5.14$ & $-4.45 \pm j 6.91$ & $-2.79 \pm j 5.18$ \\
$-4.49 \pm j 4.58$ & $-3.28 \pm j 5.61$ & $-5.91 \pm j 3.73$ & $-4.08 \pm j 7.95$ \\
$-2.7 \pm j 6.73$ & $-4.89 \pm j 7.02$ & $-2.48 \pm j 3.62$ & $-20.16 \pm j 7.11$ \\
-23.87 & $-10 \pm j 6.97$ & $-19.68 \pm j 4.4$ & $-3.33 \pm j 5.58$ \\
-19.67 & -23.33 & -19.87 & -19.713 \\
-14.16 & -19.83 & -10 & -0.2 \\
-10 & -16.99 & -10 & -20 \\
-10 & -0.26 & -0.2 & -20 \\
-0.2 & -20 & -20 & -20 \\
-20 & -20 & & \\
-20 & & & \\
\hline
\end{tabular}

TABLE 6: System eigenvalues of the coordinated designs between different UPFC-POD controllers.

\begin{tabular}{|c|c|c|c|c|c|}
\hline$m_{E} \& \delta_{E}$ & $m_{B} \& \delta_{B}$ & $m_{E} \& m_{B}$ & $\delta_{E} \& \delta_{B}$ & $m_{E} \& \delta_{B}$ & $m_{B} \& \delta_{E}$ \\
\hline$-4.71 \pm \mathrm{j} 7.04$ & $-2.85 \pm j 5.18$ & $-1.71 \pm \mathrm{j} 3.02$ & $-1.46 \pm \mathrm{j} 2.31$ & $-2.39 \pm \mathrm{j} 6.35$ & $-4.74 \pm \mathrm{j} 5.1$ \\
\hline$\zeta_{\mathrm{EM}}=0.5561$ & $\zeta_{\mathrm{EM}}=0.482$ & $\zeta_{\mathrm{EM}}=0.4927$ & $\zeta_{\mathrm{EM}}=0.5343$ & $\zeta_{\mathrm{EM}}=0.35$ & $\zeta_{\mathrm{EM}}=0.6808$ \\
\hline$-5.1 \pm j 7.33$ & $-2.71 \pm j 5.04$ & $-5.39 \pm j 10.01$ & $-1.37 \pm j 2.09$ & $-4.24 \pm j 4.93$ & $-5.7 \pm j 6.08$ \\
\hline$-10.97 \pm j 11.2$ & $-3.66 \pm j 4.51$ & $-2 \pm j 4.23$ & $-4.24 \pm j 4.93$ & $-2.96 \pm j 5.68$ & $-4.9 \pm j 5$ \\
\hline$-16.17 \pm j 2.23$ & $-10 \pm j 3.01$ & $-1.9 \pm j 3.46$ & $-2.48 \pm j 4.15$ & $-3.22 \pm j 7.25$ & $-6.29 \pm j 8.17$ \\
\hline$-5.83 \pm j 5.89$ & -24.85 & $-10 \pm j 2.13$ & $-10 \pm j 2.18$ & $-10 \pm j 4.03$ & -23.73 \\
\hline$-6.46 \pm j 8.25$ & -20.26 & -28.96 & -20.33 & -20.33 & -16.3 \\
\hline-19.87 & -19.38 & -19.87 & -19.76 & -19.76 & -19.83 \\
\hline-0.2 & -0.88 & -0.71 & -18.75 & -18.46 & -20 \\
\hline-20 & -0.36 & -0.2 & -0.2 & -0.2 & -10 \\
\hline \multirow[t]{3}{*}{-20} & -0.2 & -20 & -20 & -20 & -10 \\
\hline & -20 & -20 & -20 & -20 & -0.2 \\
\hline & -20 & & & & -20 \\
\hline
\end{tabular}

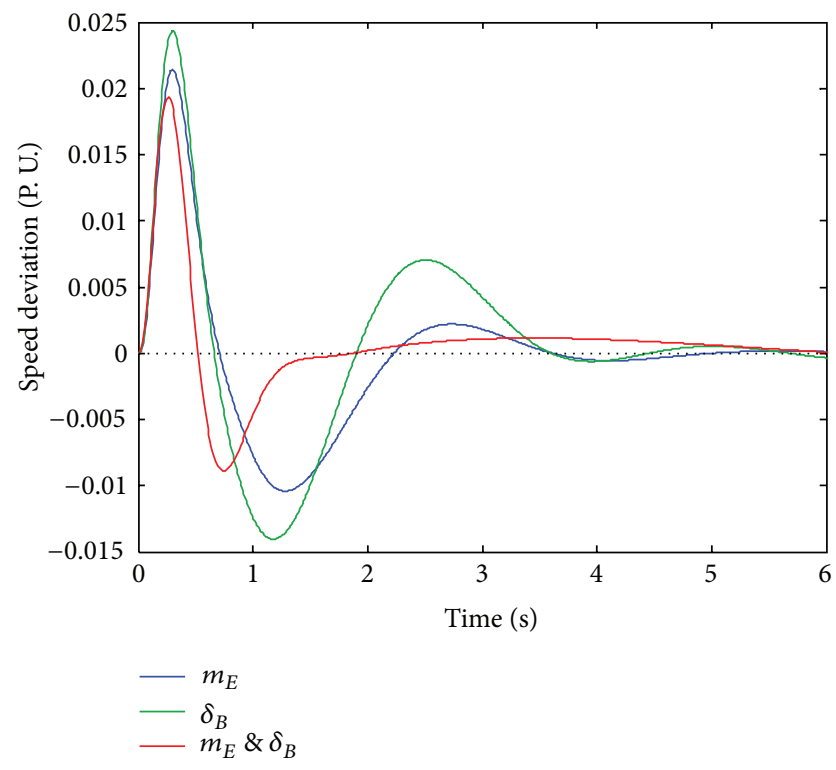

FIGURE 17: Speed variation responses for individual damping controllers $m_{E}, \delta_{B}$ and coordinated design $m_{E} \& \delta_{B}$. the best one. Finally, the coordinated designs PSS \& $m_{E}$ and (PSS \& $\left.\delta_{B}\right)$ in scheme $1 ;\left(m_{E} \& \delta_{B}\right)$ in scheme 2 provide robust damping effects over their individual control responses but no more than the best individual controls $\delta_{E}$ and $m_{B}$.

\section{Appendix}

Power system parameters (resistance and reactance are in p.u. and time constants are in second):

Generator: $M=8, \mathrm{D}=0, T_{d o}^{\prime}=5.044, X_{q}=0.6$, $X_{d}=1$ and $\grave{X}_{d}=0.3$

Excitation: $K_{a}=10, T_{a}=0.05$

Transmission line: $X_{t E}=0.1, X_{B V}=0.6$

UPFC transformers: $X_{E}=0.1, X_{B}=0.1$

Operating condition: $P_{e}=0.8 \mathrm{pu}, V_{t}=1 \mathrm{pu}$, and $V_{b}=$ $1 \mathrm{pu}$

UPFC: $m_{B}=0.0789, m_{E}=0.4013, \delta_{B}=-78.217^{\circ}$, $\delta_{E}=-85.3478^{\circ},\left(K_{S 1}-K_{S 4}\right)=1$, and $\left(T_{S 1}-T_{S 4}\right)=$ 0.05 . 
DC link parameter: $V_{\mathrm{dc}}=2 \mathrm{pu}, C_{\mathrm{dc}}=1 \mathrm{pu}$

DC voltage regulator: $K_{d P}=-5, K_{d i}=0.2$.

\section{References}

[1] P. Kundur, "Small-signal stability," in Power System Stability and Control, N. J. Balu and M. G. Lauby, Eds., pp. 699-717, McGrawHill, New York, NY, USA, 1st edition, 1994.

[2] A. Rezazadeh, M. Sedighizadeh, and A. Hasaninia, "Coordination of PSS and TCSC controller using modified particle swarm optimization algorithm to improve power system dynamic performance," Journal of Zhejiang University C, vol. 11, no. 8, pp. 645-653, 2010.

[3] B. Pal and B. Chaudhuri, Robust Control in Power Systems, Springer Science and Business Media, New York, NY, USA, 2005.

[4] P. Kumkratug, "Power system stability enhancement using unified power flow controller," American Journal of Applied Sciences, vol. 7, no. 11, pp. 1504-1508, 2010.

[5] J. H. Chow, J. J. Sanchez-Gasca, H. Ren, and S. Wang, "Power system damping controller design," IEEE Control Systems Magazine, vol. 20, no. 4, pp. 82-90, 2000.

[6] N. G. Hingorani and L. Gyugyi, Understanding Concepts and Technology of Flexible AC Transmission Systems, Wiley-IEEE Press, New York, NY, USA, 1999.

[7] L. Gyugyi, "Unified power-flow control concept for flexible AC transmission systems," IEE Proceedings C, vol. 139, no. 4, pp. 323-331, 1992.

[8] S. A. Taher and A. A. Abrishami, "UPFC location and performance analysis in deregulated power systems," Mathematical Problems in Engineering, vol. 2009, Article ID 109501, 20 pages, 2009.

[9] H. F. Wang and H. Z. Xu, "FACTS-based stabilizers to damp power system oscillations-a survey," in Proceedings of the 39th International Universities Power Engineering Conference (UPEC '04), pp. 318-322, September 2004.

[10] M. J. Gibbard, D. J. Vowles, and P. Pourbeik, "Interactions between, and effectiveness of, power system stabilizers and FACTS device stabilizers in multimachine systems," IEEE Transactions on Power Systems, vol. 15, no. 2, pp. 748-755, 2000.

[11] L. Xianzhang, E. N. Lerch, and D. Povh, "Optimization and coordination of damping controls for improving system dynamic performance," IEEE Transactions on Power Systems, vol. 16, no. 3, pp. 473-480, 2001.

[12] Y. L. Abdel-Magid and M. A. Abido, "Robust coordinated design of excitation and TCSC-based stabilizers using genetic algorithms," Electric Power Systems Research, vol. 69, no. 2-3, pp. 129-141, 2004.

[13] L. J. Cai and I. Erlich, "Simultaneous coordinated tuning of PSS and FACTS damping controllers in large power systems," IEEE Transactions on Power Systems, vol. 20, no. 1, pp. 294-300, 2005.

[14] M. A. Furini, A. L. S. Pereira, and P. B. Araujo, "Pole placement by coordinated tuning of power system stabilizers and FACTSPOD stabilizers," International Journal of Electrical Power and Energy Systems, vol. 33, no. 3, pp. 615-622, 2011.

[15] T. T. Nguyen and R. Gianto, "Neural networks for adaptive control coordination of PSSs and FACTS devices in multimachine power system," IET Generation, Transmission and Distribution, vol. 2, no. 3, pp. 355-372, 2008.

[16] A. S. P. Kanojia and B. D. V. K. Chandrakar, "Damping of power system oscillations by using coordinated tuning of POD and
PSS with STATCOM," Proceedings of World Academy of Science, Engineering and Technology, vol. 38, pp. 918-923, 2009.

[17] H. Shayeghi, A. Safari, and H. A. Shayanfar, "PSS and TCSC damping controller coordinated design using PSO in multimachine power system," Energy Conversion and Management, vol. 51, no. 12, pp. 2930-2937, 2010.

[18] E. Babaei and V. Hosseinnezhad, "A QPSO based parameters tuning of the conventional power system stabilizer," in Proceedings of the IEEE 9th International Power and Energy Conference (IPEC '10), pp. 467-471, October 2010.

[19] E. Babaei, A. M. Bolhasan, M. Sadeghi, and S. Khani, "An improved PSO and genetic algorithm based damping controller used in UPFC for power system oscillations damping," in Proceedings of the International Conference Electrical Machines and Systems (ICEMS '11), pp. 1-6, August 2011.

[20] R. Hemmati, S. M. S. Boroujeni, E. Behzadipour, and H. Delafkar, "Supplementary stabilizer design based on STATCOM," Indian Journal of Science and Technology, vol. 4, pp. 525529, 2011.

[21] R. M. Mathur and R. K. Varma, Thyristor-Based FACTS Controller for Electrical Transmission Systems, John Wiley \& Sons, Hoboken, NJ, USA, 1st edition, 2002.

[22] A. T. Al-Awami, Y. L. Abdel-Magid, and M. A. Abido, "A particle-swarm-based approach of power system stability enhancement with unified power flow controller," International Journal of Electrical Power and Energy Systems, vol. 29, no. 3, pp. 251-259, 2007.

[23] A. T. Al-Awami, M. A. Abido, and Y. L. Abdel-Magid, "Power system stability enhancement using unified power flow controllers," in Proceedings of the IEEE Grid and Cooperative Computing 5th International Conference (GCC '06), pp. 1-6, Manama, Bahrain, March 2006.

[24] H. F. Wang, "Damping function of unified power flow controller," IEE Proceedings, vol. 146, no. 1, pp. 81-87, 1999.

[25] H. Shayeghi, H. A. Shayanfar, S. Jalilzadeh, and A. Safari, "Design of output feedback UPFC controller for damping of electromechanical oscillations using PSO," Energy Conversion and Management, vol. 50, no. 10, pp. 2554-2561, 2009.

[26] F. T. S. Yao, Electric Power System Dynamics, Academic Press, New York, NY, USA, 1st edition, 1983.

[27] I. Kamwa, G. Trudel, and L. Gérin-Lajoie, "Robust design and coordination of multiple damping controllers using nonlinear constrained optimization," IEEE Transactions on Power Systems, vol. 15, no. 3, pp. 1084-1092, 2000.

[28] R. S. Parpinelli and H. S. Lopes, "New inspirations in swarm intelligence: a survey," International Journal of Bio-Inspired Computation, vol. 3, pp. 1-16, 2011.

[29] Z. Cui, F. Gao, Z. Cui, and J. Qu, "Developing a second nearestneighbor modified embedded atom method interatomic potential for lithium," Modelling and Simulation in Materials Science and Engineering, vol. 20, pp. 1-12, 2012.

[30] Z. Cui, F. Gao, Z. Cui, and J. Qu, "A second nearest-neighbor embedded atom method interatomic potential for Li-Si alloys," Journal of Power Sources, vol. 207, pp. 150-159, 2012.

[31] M. Eslami, H. Shareef, A. Mohamed, and M. Khajehzadeh, "Coordinated design of PSS and SVC damping controller using CPSO," in Proceedings of the 5th International Power Engineering and Optimization Conference (PEOCO '11), pp. 11-16, Selangor, Malaysia, June 2011. 


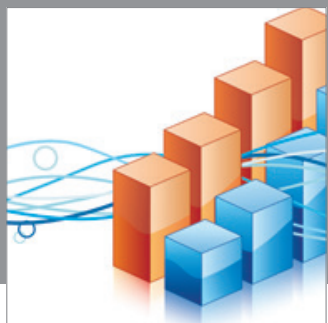

Advances in

Operations Research

mansans

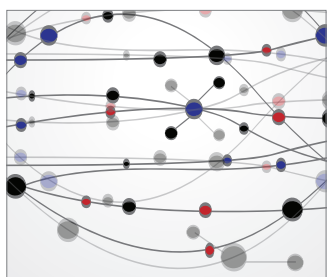

The Scientific World Journal
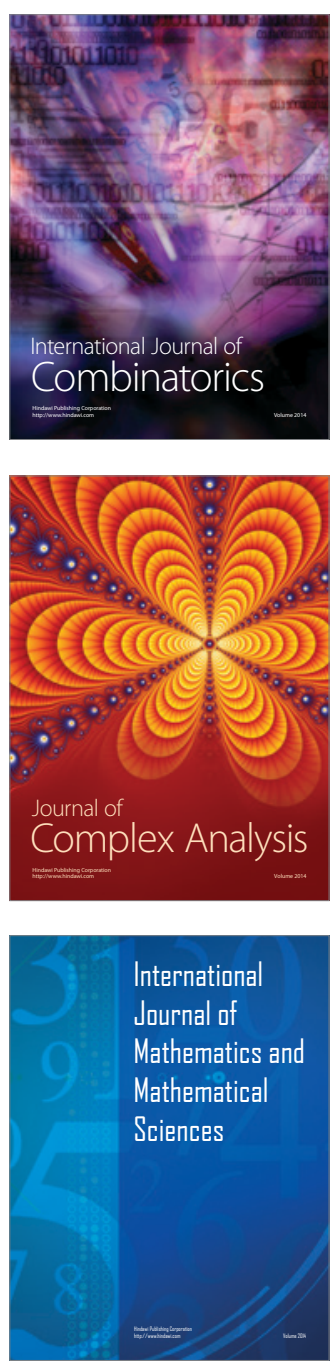
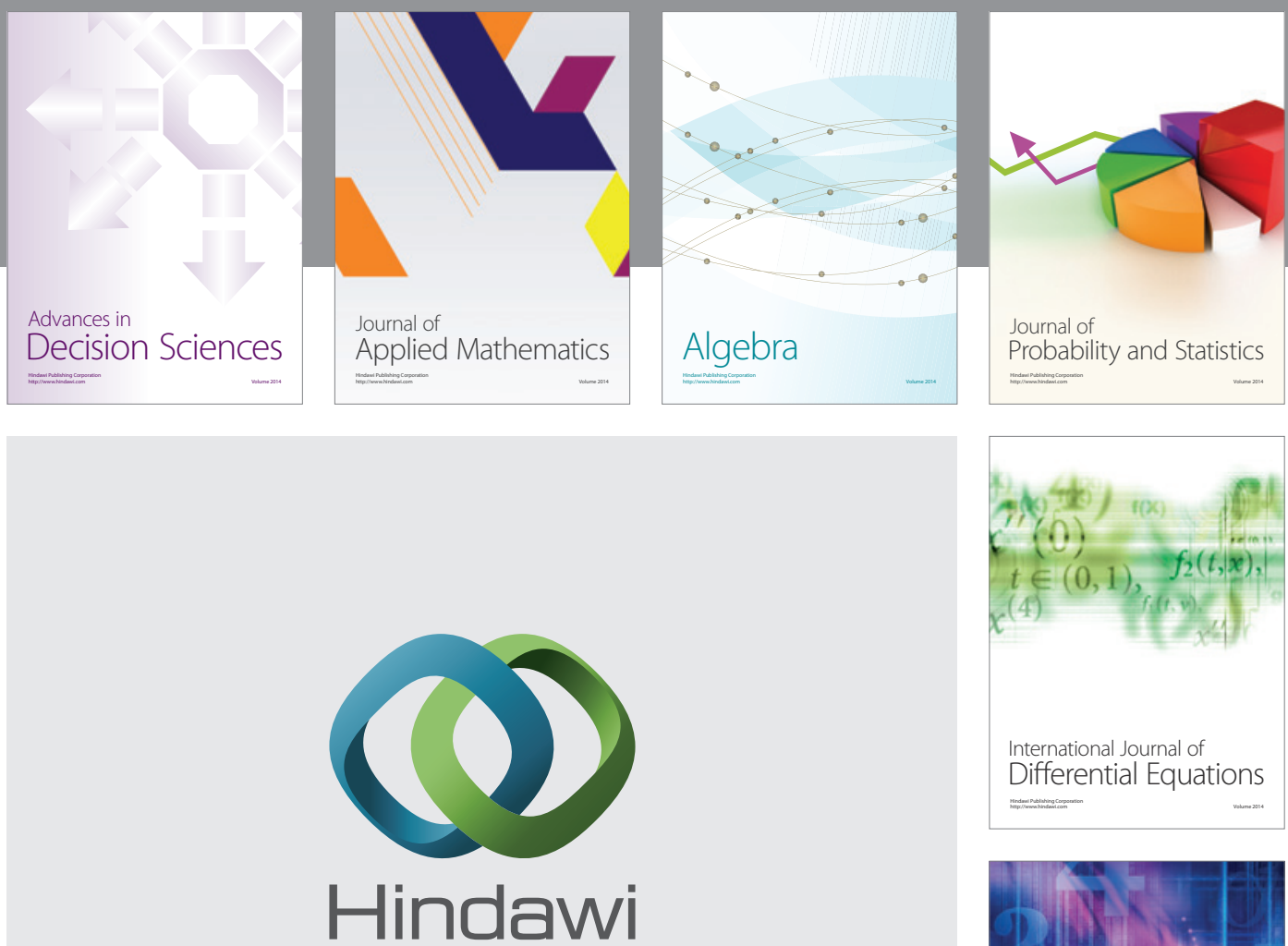

Submit your manuscripts at http://www.hindawi.com
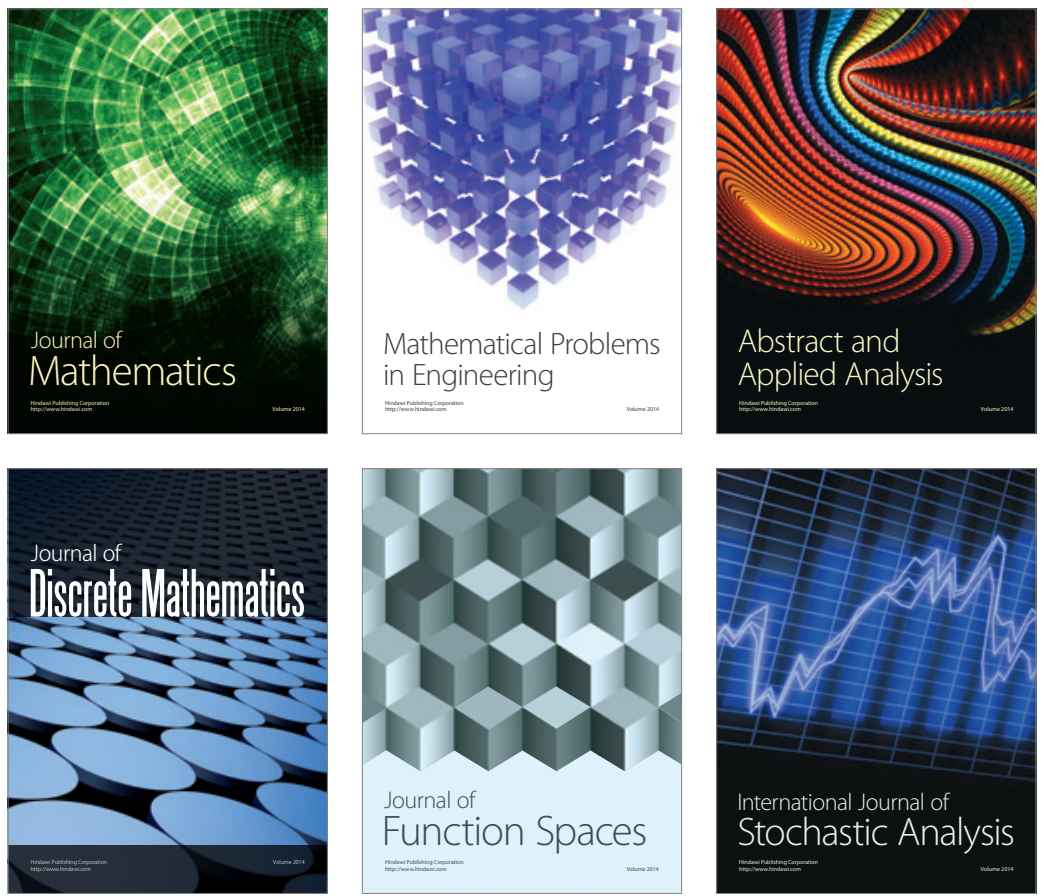

Journal of

Function Spaces

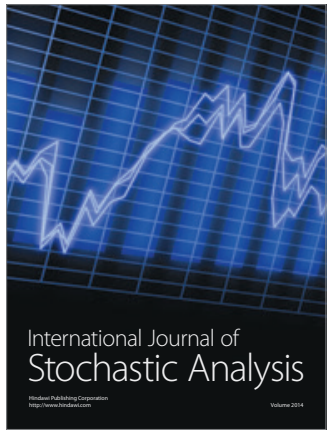

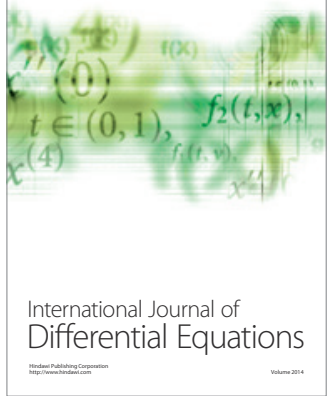
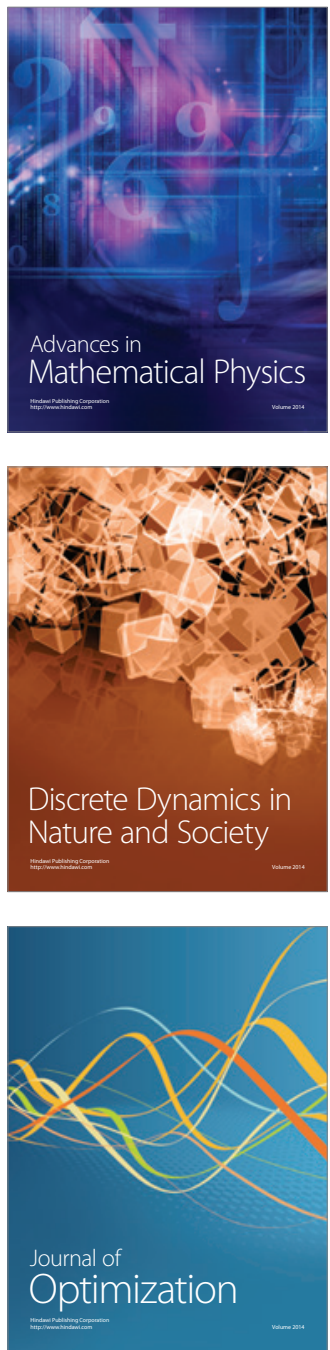